\title{
Multiplicative-noise Can Suppress Chaotic Oscillation in Lotka-Volterra Type Competitive Model
}

\author{
P.S. Mandal, M. Banerjee* \\ Department of Mathematics and Statistics \\ Indian Institute of Technology, Kanpur \\ Kanpur - 208016, INDIA
}

\begin{abstract}
Recently, Wang and Xiao studied a four-dimensional competitive Lotka-Volterra system within a deterministic environment in [11]. With the help of numerical example they showed the existence of a chaotic attractor through the period doubling route. In this paper, we are interested to study the dynamics of the same model in presence of environmental driving forces. To incorporate the environmental driving force into the deterministic system, we perturb the growth rates of each species by white noise terms. Then we prove that the unique positive global solution exists for the noise added system and the general $p$-th order moment of it is bounded for $p \geq 1$, which ensures that the solution is stochastically bounded. It is also shown that the solution of the stochastic system is stochastically permanent under some simple conditions. Finally, we demonstrate the noise induced oscillation for the concerned model with the help of numerical example..
\end{abstract}

Keywords and phrases: competition, bifurcation, chaos, noise, moment, stochastic permanence

Mathematics Subject Classification: 34C23, 60J25, 65P20, 92D25

\section{Introduction}

Ecological systems are biological communities of interacting organisms exposed within natural environment. Growth and evolution of each organism and species are affected by the fluctuations in environmental conditions to some extent. The intrinsic nonlinearities involved with the interactions between individuals and environmental driving forces are the main reasons behind the complex dynamical behavior of ecological systems, which sometimes very sensitive to initial conditions, small amount of external perturbations and to the small amplitude fluctuation in environmental conditions [1]. Quite a good number of models have been considered and analyzed thoroughly by several researchers to study the complex dynamics of interacting populations (some relevant texts are [2-5]).

*Corresponding author. E-mail: malayb@iitk.ac.in 
The simplest and most well known mathematical model for population interactions are Lotka-Volterra type models. The general Lotka-Volterra model which has become the starting point for a wide variety of mathematical models in biology, specially in ecology was originally derived by Volterra to describe the interaction between a predator species and a prey species [8] and independently by Lotka to describe a chemical reaction [9]. These models are widely used in mathematical modelling of natural processes which exhibit periodic fluctuations but the models with deterministic setup usually have stationary steady-states or periodic solutions. Recently some researchers have been extensively explored the instances where chaos is observed for Lotka-Volterra type competition models [10,11].

The general $n$-species competitive Lotka-Volterra system is described by the following system of $n$ coupled non-linear ordinary differential equations

$$
\frac{d x_{i}(t)}{d t}=x_{i}\left(b_{i}-\sum_{j=1}^{n} a_{i j} x_{j}(t)\right), \quad i=1, \ldots, n,
$$

subjected to positive initial conditions $x_{i}(0)>0,(1 \leq i \leq n)$, where $x_{i}(t)$ is the number of individuals or population density of the $i$-th population at any instant of time $t, b_{i}$ denotes the intrinsic growth rate of the $i$-th species and $a_{i j}$ 's $(1 \leq i, j \leq n)$ are the measure of the extent to which the $j$-th species affects the growth rate of the $i$-th species. $b_{i}>0, a_{i j}>0$ for all $1 \leq i, j \leq n$ according to the definition of $n$-dimensional competitive Lotka-Volterra system [12]. In [6], Smale has demonstrated that for $n \geq 5$, one can find competitive Lotka-Volterra systems exhibiting structurally stable strange attractors for certain range of parameter values. In [13-15], Hirsch has demonstrated that for an $n$-dimensional competitive Lotka-Volterra system on $\mathbb{R}_{+}^{n}$, there exists an invariant surface $S$ which is homeomorphic to the standard $(n-1)$ dimensional unit simplex $\Delta_{n-1}=\left\{x_{i}: x_{i} \geq 0, \sum_{i=1}^{n} x_{i}=1\right\}$. It is also a global attractor for trajectories starting from every point (excluding the origin) in the domain $D=\left\{x_{i}: x_{i} \geq 0\right\}$ and carries all the asymptotic dynamical features of equation (1.1). The surface $S$ is known as carrying simplex, the term was introduced by Zeeman[16]. Hence for $n=2$, the possible dynamical behavior of system (1.1) is trivial. For a three dimensional competitive system, one can only get the stable coexisting equilibrium point and periodic orbits as compact indecomposable invariant sets using Poincare-Bendixson theorem [17-22]. So the dynamics of system (1.1) is simple for $n=3$. But the possible dynamics of system (1.1) may not be always simple for $n \geq 4$ (see [23,24] and references cited there in for more details). In [11], authors has showed that for $n=4$, system (1.1) exhibit chaotic behavior as Smale claimed in [6] and also they explored the mechanism of occurrence of chaotic behavior. The authors have carried out the complete bifurcation analysis and demonstrated the period doubling route to chaos. Now the question is, whether chaotic oscillation in population density of one or more interacting species supported by any realistic data or not. In [7], Ellner and Turchin described a methodology to analyze short time series and demonstrated the presence of deterministic chaos by analyzing a short and noisy time series. There are more results in support of chaotic dynamics exhibited by interacting populations [25-28].

The population size of any species involved with any ecosystem fluctuates continuously due to the random variation in external driving forces like temperature, intensity of light, fluctuation in available nutrient levels and so on [29]. Modeling population dynamics within random environment provides the key way to understand this kind of fluctuation in population sizes. In [2], May has demonstrated the formulation of stochastic model by perturbing deterministic rate constants by white noise terms and observed that a larger deviation from equilibrium density is possible and leading to stochastic instability (the deviation from mean population increases) due to large magnitude of environmental forcing. Since the general qualitative response of terrestrial and aquatic ecosystems under environmental fluctuation could be inherently different, the outcomes of the noise added systems solely depend on the character of the physical frequency distributions of noise under consideration. In case of terrestrial systems, the environmental variability is large over shorter and longer time periods and can be expected to develop internal mechanisms, which could cope with short-term variability and minimize the effects of long-term 
variations. Hence using white noise terms, one can get better outcomes for terrestrial ecosystems. For aquatic ecological systems, colored noise give the better results instead of white noise as compared to terrestrial systems $[30,31]$. Also for the aquatic system, minimum robust internal processes are required to handle the smaller amplitude variability over short time periods which commensurate with the life span of the aquatic micro-organisms. Generally environmental fluctuations affect the dynamical evolution of interacting populations in two different ways. When environmental fluctuations affect the growth rates of various species then it can be modeled by perturbing the growth rates by white noise terms. On the other hand, due to random fluctuation in environmental conditions the several intrinsic factors, namely, growth rate, death rate, rate of predation, rate of immigration and emigration etc. To model these type of phenomena we take help of multiplicative noise terms introduced in to the growth equations of concerned species. A brief and concise discussion in this context is available in [32]. Analytical results obtained through noise added models strongly suggest that most ecological systems are not chaotic [7], but there are very limited validations of this theoretical claim [33].

Researchers have mainly been drawn their attention towards the dynamical consequences of population interactions based upon the deterministic setup and often ignoring the effects of environmental variability. However, the role of environmental driving forces has recently been considered in theoretical ecology. A wide range of dynamical analysis of interacting populations with deterministic setup is based upon the assumption that stochastic deviations are small enough for large population [34]. But in reality, the interaction of the environmental driving forces with the nonlinearity involved with the system has given rise to new counterintuitive phenomena, like, stochastic resonance [35], noise-enhanced stability [36], noise-induced non-equilibrium transitions [37], noise-induced multistability and non-equilibrium phase transitions $[38,39]$. Although the dynamics of ecological systems in presence of environmental noises are less studied where the basic deterministic model exhibit chaotic oscillations.

In the present paper, we recall the period doubling route to chaos for a four dimensional Lotka-Volterra type competitive model in section 2. In section 3, we formulate the stochastic model by incorporating white noise terms into the intrinsic growth rates of four competitors and then prove the existence of global solution for the stochastic model. The proof for boundedness of $p$-th order moments for every species and ultimate boundedness of solutions for the stochastic model is also provided in section 3 . In section 4 , we derive the parametric restrictions required for the stochastic permanence implying the coexistence of all species at all future time. The analytical results obtained are verified with the help of numerical experiments in section 5. Noise enhanced oscillation and non-equilibrium fluctuations in presence of high magnitude environmental driving forces are discussed in section 5. Ecological interpretations of obtained results are provided in the concluding section.

\section{The deterministic Model}

In this paper, we consider the following four dimensional competitive Lotka-Volterra model

$$
\frac{d y_{i}}{d t}=y_{i}\left(\rho_{i}-\sum_{j=1}^{4} \alpha_{i j} y_{j}\right), \quad 1 \leq i \leq 4,
$$

subjected to the positive initial conditions $y_{i}(0)=y_{i 0}>0,1 \leq i \leq 4$. This model is studied extensively by Wang and Xiao [11], mainly with the help of numerical investigations, and here we recall basic results without any proof. These results are required to identify the coexisting equilibrium point and calculation of Hopf-bifurcation threshold.

Using the linear transformation $x_{i}=\frac{\alpha_{i i}}{\rho_{i}} y_{i}$, system (2.1) can be transformed as follows

$$
\frac{d x_{i}}{d t}=\rho_{i} x_{i}\left(1-\sum_{j=1}^{4} \alpha_{i j} x_{j}\right), \quad 1 \leq i \leq 4,
$$


where $\alpha_{i i}=1$ for $i=1,2,3,4$. Coexisting equilibrium point of model (2.2) is the unique point of interaction of four hyperplanes (given by $\sum_{j=1}^{4} \alpha_{i j} x_{j}=1,1 \leq i \leq 4$ ) and let us denote the unique interior equilibrium point by $\left(p_{1}, p_{2}, p_{3}, p_{4}\right)$. For simplicity of algebraic calculations, the equilibrium point $\left(p_{1}, p_{2}, p_{3}, p_{4}\right)$ can be translated to $(1,1,1,1)$ using the following transformation

$$
z_{i}=\frac{x_{i}}{p_{i}}, i=1,2,3,4 \text {. }
$$

Substituting $x_{i}=p_{i} z_{i}, i=1,2,3,4$, in $(2.2)$ we get,

$$
\frac{d z_{i}}{d t}=z_{i}\left(\sum_{j=1}^{4} c_{i j} p_{j}\left(1-z_{j}\right)\right), \quad i=1,2,3,4,
$$

where $c_{i j}=\rho_{i} \alpha_{i j}$ for $1 \leq i, j \leq 4$. For local asymptotic stability of interior equilibrium point $E_{*}(1,1,1,1)$ and existence of Hopf-bifurcating periodic solution around it, we have to study the nature of the eigenvalues of the Jacobian matrix for system (2.3) evaluated at interior equilibrium point. For this purpose we define the following matrices,

$$
\begin{gathered}
-J=-\left(J_{i j}\right)_{4 \times 4}=-\left(\begin{array}{llll}
c_{11} p_{1} & c_{12} p_{2} & c_{13} p_{3} & c_{14} p_{4} \\
c_{21} p_{1} & c_{22} p_{2} & c_{23} p_{3} & c_{24} p_{4} \\
c_{31} p_{1} & c_{32} p_{2} & c_{33} p_{3} & c_{34} p_{4} \\
c_{41} p_{1} & c_{42} p_{2} & c_{43} p_{3} & c_{44} p_{4}
\end{array}\right) \\
M_{i j}=\operatorname{det}\left(\begin{array}{cc}
J_{i i} & J_{i j} \\
J_{j i} & J_{j j}
\end{array}\right), M_{i j k}=\operatorname{det}\left(\begin{array}{ccc}
J_{i i} & J_{i j} & J_{i k} \\
J_{j i} & J_{j j} & J_{j k} \\
J_{k i} & J_{k j} & J_{k k}
\end{array}\right) .
\end{gathered}
$$

The characteristic equation of the Jacobian matrix evaluated at $E_{*}$ is given by

$$
\lambda^{4}+\left(\sum_{i=1}^{4} J_{i i}\right) \lambda^{3}+\left(\sum_{1 \leq i<j \leq 4} M_{i j}\right) \lambda^{2}+\left(\sum_{1 \leq i<j<k \leq 4} M_{i j k}\right) \lambda+\operatorname{Det}(J)=0 .
$$

Using Routh-Hurwitz criteria, the local asymptotic stability of $E_{*}$ demands the satisfaction of the following conditions

$$
\begin{gathered}
\sum_{i=1}^{4} J_{i i}>0, \quad \sum_{1 \leq i<j<k \leq 4} M_{i j k}>0, \quad \operatorname{Det}(J)>0, \\
\sum_{i=1}^{4} J_{i i} \sum_{1 \leq i<j \leq 4} M_{i j} \sum_{1 \leq i<j<k \leq 4} M_{i j k}>\left(\sum_{1 \leq i<j<k \leq 4} M_{i j k}\right)^{2}+\left(\sum_{i=1}^{4} J_{i i}\right)^{2} \operatorname{Det}(J) .
\end{gathered}
$$

For the model under consideration, it is difficult to find explicit criteria in terms of system parameters for the local asymptotic stability of $E_{*}$, but it can be verified with the help of numerical example. Now we recall the Hopf-bifurcation criteria for model (2.3), as $E_{*}$ becomes unstable through Hopf-bifurcation and small amplitude periodic solution bifurcates from the stable interior equilibrium point [11]. Here we state the following lemma from [11], regarding the nature of eigenvalues of the Jacobian matrix.

Lemma 2.1 (i) The necessary and sufficient conditions for which the Jacobian matrix of system (2.3) evaluated at equilibrium $(1,1,1,1)$ has a negative real eigenvalue, a real non-zero eigenvalue and a pair of purely imaginary eigenvalues are $\operatorname{Det}(J) \neq 0, \sum_{i=1}^{4} J_{i i}>0, \sum_{1 \leq i<j<k \leq 4} M_{i j k}>0$, and

$$
\left(\sum_{1 \leq i, j \leq 4} M_{i j}-\frac{\sum_{1 \leq i<j<k \leq 4} M_{i j k}}{\sum_{i=1}^{4} J_{i i}}\right) \times\left(\frac{\sum_{1 \leq i<j<k \leq 4} M_{i j k}}{\sum_{i=1}^{4} J_{i i}}\right)=\operatorname{Det}(J) .
$$


(ii) The necessary and sufficient conditions for which the Jacobi matrix of system (2.3) at equilibrium (1, $1,1,1)$ has a negative real eigenvalue, a zero eigenvalue and a pair of purely imaginary eigenvalues are $\operatorname{tr}(J)>0, \sum_{1 \leq i, j \leq 4} M_{i j}>0, \operatorname{Det}(J)=0$ and

$$
\sum_{i=1}^{4} J_{i i} \sum_{1 \leq i, j \leq 4} M_{i j}=\sum_{1 \leq i<j<k \leq 4} M_{i j k} .
$$

moreover the eigenvalues are $0,-\operatorname{tr}(J)$ and $\pm i \sqrt{\sum_{1 \leq i, j \leq 4} M_{i j}}$.

Based upon above lemma, we can state the Hopf-bifurcation theorem for the system (2.3) around the interior equilibrium point $E_{*}$ as follows,

Theorem 2.1 Suppose that system (2.2) has a unique positive equilibrium $E^{*}=\left(p_{1}, p_{2}, p_{3}, p_{4}\right)$. Then the equilibrium $E^{*}$ is non-degenerate and system (2.2) undergoes into Hopf-bifurcation around $E^{*}$ whenever the conditions $\sum_{i=1}^{4} J_{i i}>0, \sum_{1 \leq i<j<k \leq 4} M_{i j k}>0$ and condition (2.7) are satisfied.

Now we consider the following 4 -dimensional competitive Lotka-Volterra system with the specific choice of parameter values

$$
\begin{aligned}
& \frac{d x_{1}}{d t}=x_{1}\left(1-x_{1}-0.06 s x_{2}-2.2 s x_{3}-0.5 s x_{4}\right), \\
& \frac{d x_{2}}{d t}=0.42 x_{2}\left(1-0.36 s x_{1}-x_{2}-0.05 s x_{3}-1.32 s x_{4}\right), \\
& \frac{d x_{3}}{d t}=0.57 x_{3}\left(1-1.41 s x_{1}-1.07 s x_{2}-x_{3}-0.17 s x_{4}\right), \\
& \frac{d x_{4}}{d t}=0.72 x_{4}\left(1-0.26 s x_{1}-0.54 s x_{2}-1.15 s x_{3}-x_{4}\right),
\end{aligned}
$$

where $s$ is the bifurcation parameter. The same example is investigated in details by Wang and Xiao [11], here we recall the main features briefly. These results will help us to understand the effect of environmental noise on the four dimensional competitive system. Interior equilibrium point $E_{*}$ is feasible whenever $.7<s$. For $0.7<s<s_{1}$, the unique interior equilibrium point of above system is locally asymptotically stable where $s_{1} \approx 0.7965$ is the Hopf-bifurcation threshold. Fig. 1 shows the convergence of four species to their respective steady-state values. The limit cycle bifurcating from the interior equilibrium point remain stable whenever $s_{1}<s<s_{2} \approx 0.92915$. Fig. 2 depicts the stationary oscillatory population growth after ignoring initial transients along with the projection of limit cycle on $x_{1} x_{2}$-plane. At $s_{2} \approx 0.92915$ first period doubling occurs and then system undergoes successive period doubling with the increase in magnitude of $s$. 2-periodic and 4-periodic solutions are presented in Fig. 3 and Fig. 4 respectively, values of the bifurcation parameter are mentioned in the caption of the figures. The competitive system is capable to exhibit chaotic co-existence for values of $s>1$. Chaotic time series is presented in Fig. 5 for $s=1.1$. In order to understand the shape of chaotic attractor, its projections on all possible two dimensional planes are given in Fig. 6.

\section{Stochastic Model}

Throughout the discussion in previous section, we dealt with the assumption that the parameters involved with the model system are all constants irrespective of time and environmental influences. In realistic situation, all intrinsic parameters exhibit random variations to a greater or lesser extent due to continuous fluctuation in the environmental conditions. In the last section, we have briefly discussed about the stability of coexisting steady state and how this stability breaks down with the change of control parameter $s$ and ultimately leads to chaotic dynamics through the period doubling route. In this section, we consider the effect of environmental fluctuation on the 4-dimensional competitive model (2.2). 


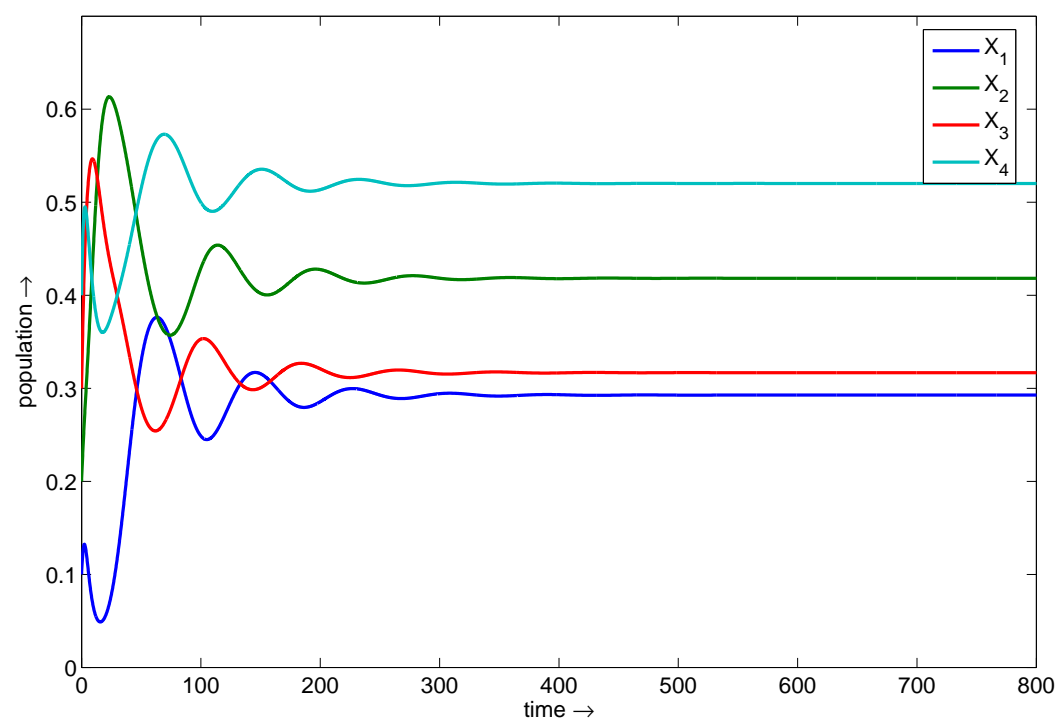

FiguRE 1. Each species going to deterministic steady-state after some initial transient oscillations. Numerical simulation results are obtained for the deterministic model (2.8) - (2.11) with $s=.75$.
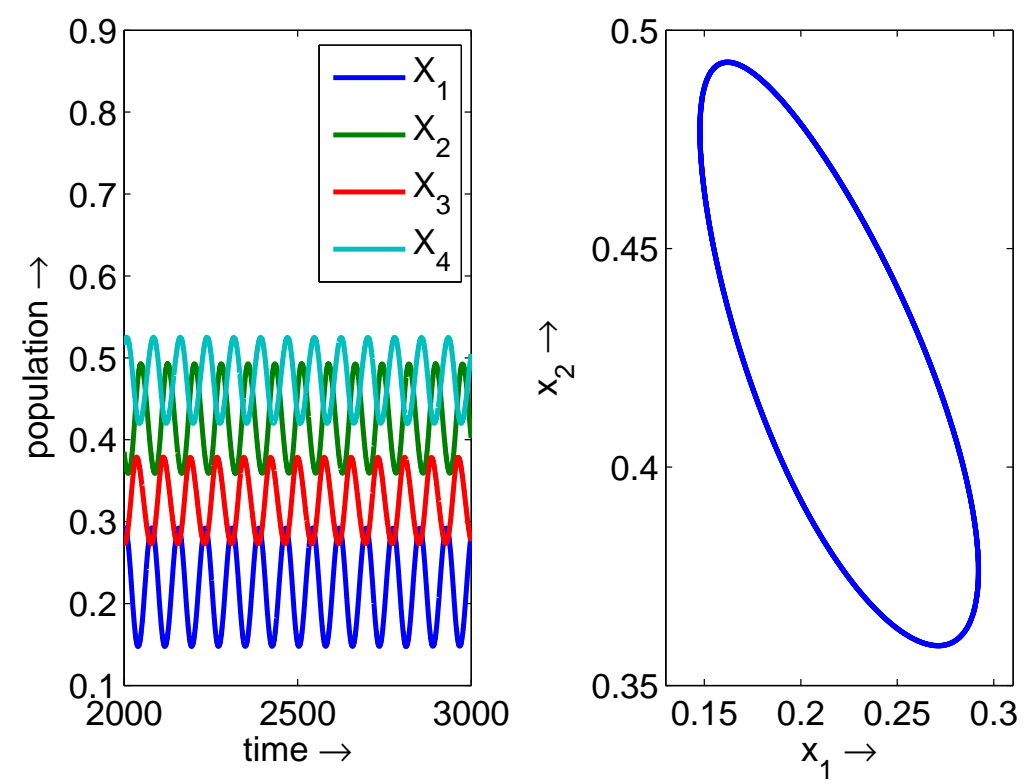

Figure 2. Hopf-bifurcation periodic solution obtained for $s=.9$ (left panel) and the projection of limit-cycle on $x_{1} x_{2}$-plane (right panel). 

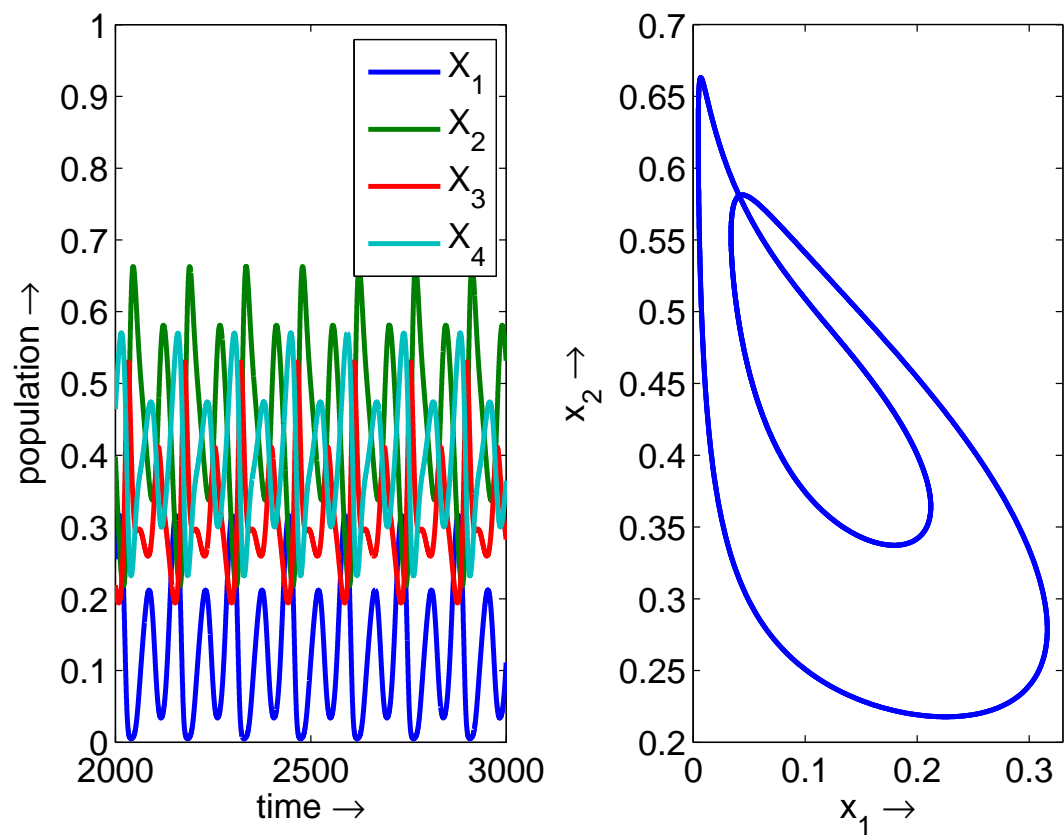

Figure 3. Two periodic solution is obtained for $s=.95$.
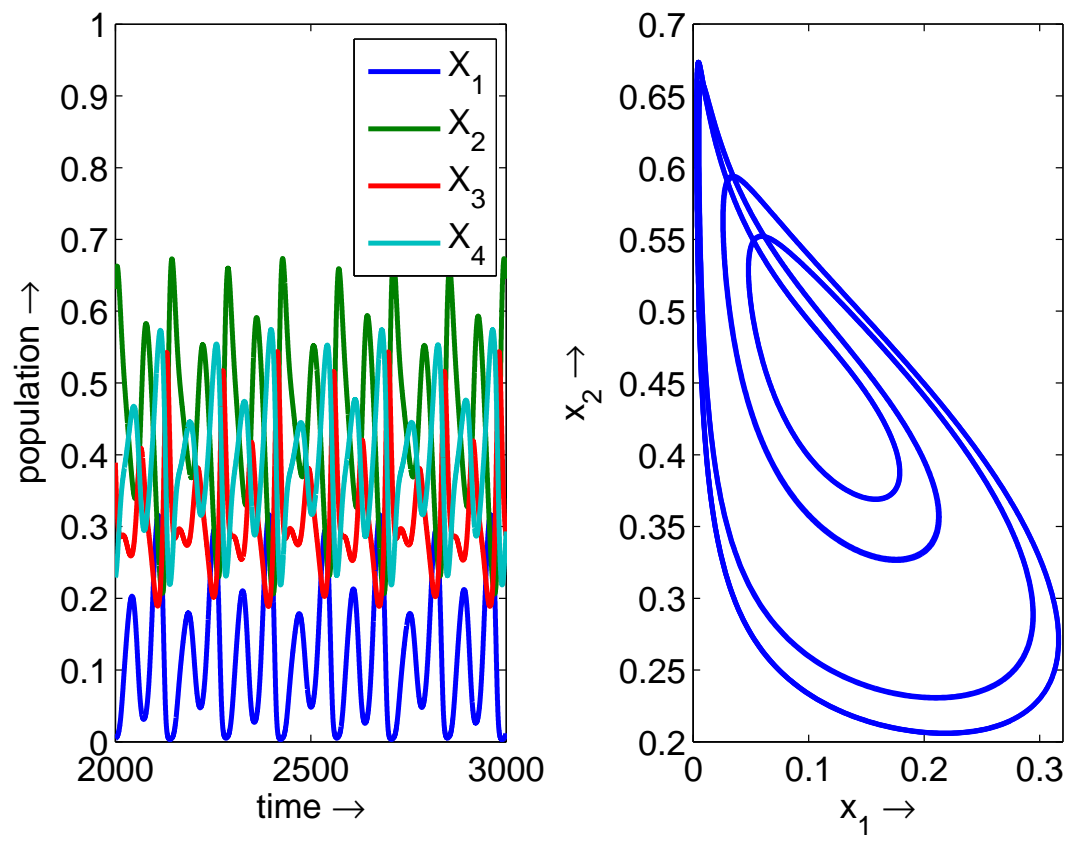

FiguRE 4. Time evolution of each species when the period of oscillatory solution is four (left panel) and projection of 4-periodic solution on $x_{1} x_{2}$-plane.Numerical simulation is performed with $s=.965$. 


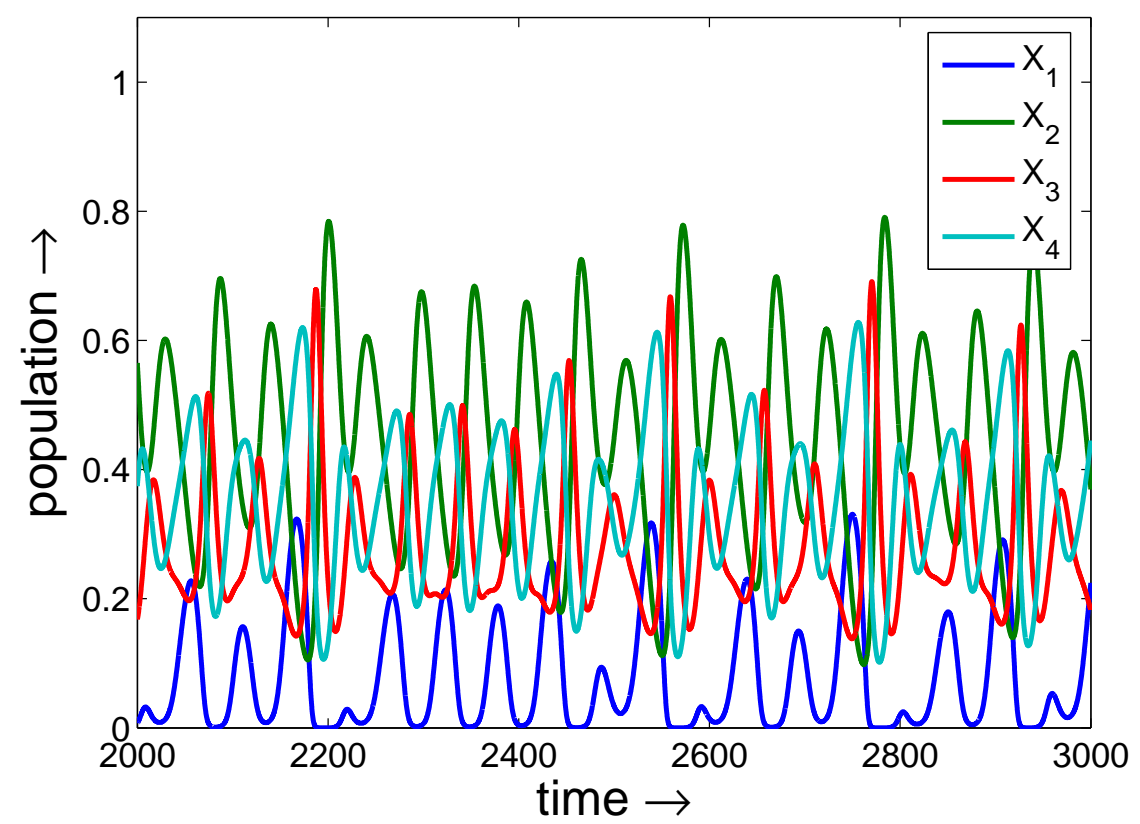

FiguRE 5. Chaotic oscillation is observed for $s>1$ and numerical results reported here is obtained for $s=1.1$.
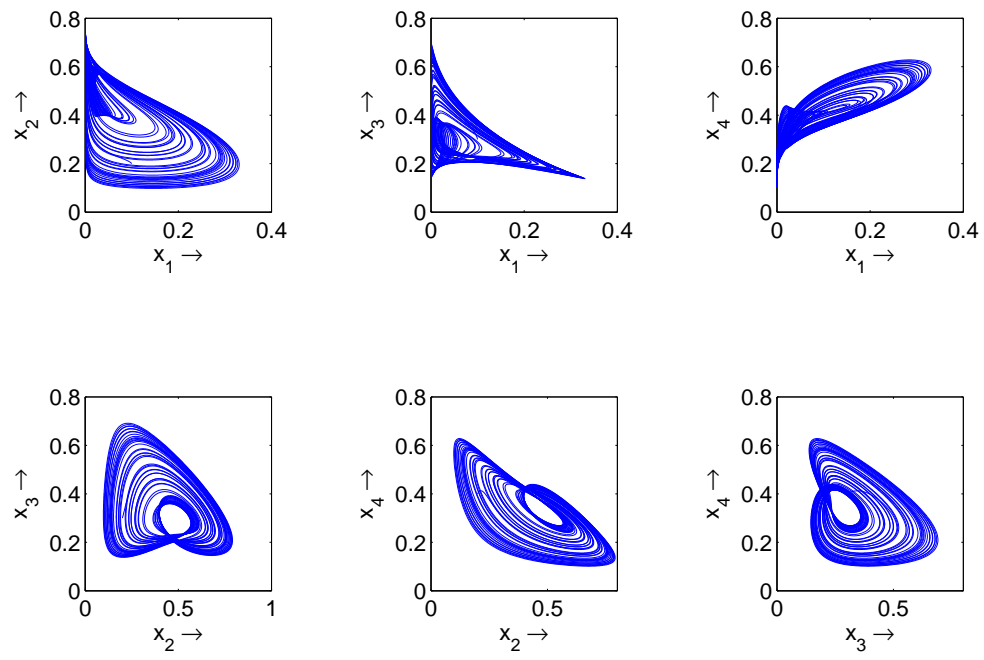

FiguRE 6. Projections of chaotic attractor on each of the two dimensional coordinate planes, demonstrating the shape of the attractor corresponding to the time series presented in Fig. 5. 
Environmental fluctuation is one of the most important component in an ecosystem and one can not overlook this aspect during the modeling and analysis of a realistic ecological system exposed within the open environment. Stability of equilibrium point for deterministic model imply that all population will remain at a fixed state at all future time. Most of the natural phenomena randomly fluctuate around some average value instead of remaining at a deterministic fixed level. Hence the stable deterministic equilibrium scenario is unable to capture the realistic feature [40,41]. May [2] pointed out that birth rates, carrying capacities, competition coefficients and other parameters which characterize the natural biological system exhibit random fluctuations due to the continuous random change in the environmental conditions. Elton [42] observed that fluctuations occur in animal numbers due to the instability of the environment. For the models within deterministic setup, we always seek the constant equilibrium population and then investigate its stability which follows from the dynamics of the interactions between and within the species. Based upon the nonlinearity involved with the model and the specific choice of parameter values, we observe oscillatory, quasi-periodic or chaotic oscillations but they are all deterministic in nature. In a stochastic system, it is impossible to find out an equilibrium point, but it is reasonable to search for the probabilistic 'smoke cloud', described by the stationary probability distribution. For the stochastic model system governed by the system of SDEs, there is a continuous spectrum of disturbances generated by the environmental stochasticity and the system is in tension between two countervailing tendencies. The random environmental driving forces are responsible to spread dense cloud and supports the probability distribution to diffuse, while the dynamics of stabilizing population interactions tend to restore the populations to their mean value in order to compact the cloud [2]. Model systems consisting of compact cloud of population distributions are generally called stochastically stable systems.

To study the effect of the environmental fluctuation, we have to formulate the stochastic system corresponding to the deterministic one by incorporating the environmental driving forces. This kind of stochastic model is known as noise-added/noise-driven system and this kind of modeling approach is capable to capture the random change in net growth rate of all populations.

To take into account the effect of randomly fluctuating environment, we perturb the intrinsic growth rates of each species by additive white noise terms to obtain the noise added model from the deterministic system (2.2). Therefore the noise added model is given by the following system of equations

$$
\begin{aligned}
& \frac{d x_{1}(t)}{d t}=\rho_{1} x_{1}(t)\left(1-x_{1}(t)-\alpha_{12} x_{2}(t)-\alpha_{13} x_{3}(t)-\alpha_{14} x_{4}(t)\right)+\sigma_{1} x_{1} \xi_{1}(t), \\
& \frac{d x_{2}(t)}{d t}=\rho_{2} x_{2}(t)\left(1-\alpha_{21} x_{1}(t)-x_{2}(t)-\alpha_{23} x_{3}(t)-\alpha_{24} x_{4}(t)\right)+\sigma_{2} x_{2}(t) \xi_{2}(t), \\
& \frac{d x_{3}(t)}{d t}=\rho_{3} x_{3}(t)\left(1-\alpha_{31} x_{1}(t)-\alpha_{32} x_{2}(t)-x_{3}(t)-\alpha_{34} x_{4}(t)\right)+\sigma_{3} x_{3}(t) \xi_{3}(t), \\
& \frac{d x_{4}(t)}{d t}=\rho_{4} x_{4}(t)\left(1-\alpha_{41} x_{1}(t)-\alpha_{42} x_{2}(t)-\alpha_{43} x_{3}(t)-x_{4}(t)\right)+\sigma_{4} x_{4}(t) \xi_{4}(t),
\end{aligned}
$$

subjected to the initial conditions $x_{1}(0) \equiv x_{10}>0, x_{2}(0) \equiv x_{20}>0, x_{3}(0) \equiv x_{30}>0, x_{4}(0) \equiv x_{40}>0$. $\xi_{1}(t), \xi_{2}(t), \xi_{3}(t)$ and $\xi_{4}(t)$ are four mutually independent white noise terms, characterized by $\left\langle\xi_{1}(t)\right\rangle=$ $\left\langle\xi_{2}(t)\right\rangle=\left\langle\xi_{3}(t)\right\rangle=\left\langle\xi_{4}(t)\right\rangle=0$ and $\left\langle\xi_{i}(t) \xi_{j}\left(t_{1}\right)\right\rangle=\delta_{i j} \delta\left(t-t_{1}\right)$ where $\delta_{i j}$ is Kronecker delta and $\delta($.$) is$ the 'Dirac- $\delta$ ' function [43-45]. Here parameters $\sigma_{1}, \sigma_{2}, \sigma_{3}$ and $\sigma_{4}$ denote the intensities of environmental driving forces. The above model can be written into the following standard form of stochastic differential equations:

$$
\begin{aligned}
& d x_{1}(t)=\rho_{1} x_{1}(t)\left(1-x_{1}(t)-\alpha_{12} x_{2}(t)-\alpha_{13} x_{3}(t)-\alpha_{14} x_{4}(t)\right) d t+\sigma_{1} x_{1}(t) d B_{1}(t), \\
& d x_{2}(t)=\rho_{2} x_{2}(t)\left(1-\alpha_{21} x_{1}(t)-x_{2}(t)-\alpha_{23} x_{3}(t)-\alpha_{24} x_{4}(t)\right) d t+\sigma_{2} x_{2}(t) d B_{2}(t), \\
& d x_{3}(t)=\rho_{3} x_{3}(t)\left(1-\alpha_{31} x_{1}(t)-\alpha_{32} x_{2}(t)-x_{3}(t)-\alpha_{34} x_{4}(t)\right) d t+\sigma_{3} x_{3}(t) d B_{3}(t), \\
& d x_{4}(t)=\rho_{4} x_{4}(t)\left(1-\alpha_{41} x_{1}(t)-\alpha_{42} x_{2}(t)-\alpha_{43} x_{3}(t)-x_{4}(t)\right) d t+\sigma_{4} x_{4}(t) d B_{4}(t),
\end{aligned}
$$

where $B_{i}(t), \quad i=1,2,3,4$ are one-dimensional Wiener processes independent of each other [46] and defined over the complete probability space $(\Omega, \mathcal{F}, P)$ with a filtration $\left\{\mathcal{F}_{t}\right\}_{t \geq 0}$ satisfying the usual con- 
ditions (i.e. it is increasing and right continuous while $\mathcal{F}_{0}$ contains all $P$-null sets [47]). The relation between the white noise terms and Wiener processes are described by $d B_{r}=\xi_{r}(t) d t, r=1,2,3,4$ [48]. White noise is a pathwise derivatives of Wiener processes. In the rest of this paper, we consider (3.5)-(3.8) as an Itô stochastic differential system of the following form

$$
d X_{t}=f\left(t, X_{t}\right) d t+g\left(t, X_{t}\right) d B(t), X_{t 0} \equiv X_{0},
$$

where solution $\left(X_{t}, t>0\right)$ is an Itô process [49,50], ' $f$ ' is slowly varying continuous component or drift term, ' $g$ ' is rapidly varying continuous random component or diffusion term and $B(t)$ is a fourdimensional stochastic process having scalar Wiener process as components, the increments $\Delta B_{j}(t)=$ $B_{j}(t+\Delta t)-B_{j}(t)$ are independent Gaussian random variables $N(0, \Delta t)$. In $(3.9)$,

$$
\begin{gathered}
X_{t} \equiv\left(x_{1}, x_{2}, x_{3}, x_{4}\right)^{T}, \quad B(t) \equiv\left(B_{1}(t), B_{2}(t), B_{3}(t), B_{4}(t)\right)^{T}, \\
f \equiv\left(\begin{array}{l}
F_{1}\left(x_{1}, x_{2}, x_{3}, x_{4}\right) \\
F_{2}\left(x_{1}, x_{2}, x_{3}, x_{4}\right) \\
F_{3}\left(x_{1}, x_{2}, x_{3}, x_{4}\right) \\
F_{4}\left(x_{1}, x_{2}, x_{3}, x_{4}\right)
\end{array}\right), \quad g \equiv\left(\begin{array}{cccc}
\sigma_{1} x_{1} & 0 & 0 & 0 \\
0 & \sigma_{2} x_{2} & 0 & 0 \\
0 & 0 & \sigma_{3} x_{3} & 0 \\
0 & 0 & 0 & \sigma_{4} x_{4}
\end{array}\right),
\end{gathered}
$$

where

$$
F_{i}\left(X_{i}\right)=\rho_{i} x_{i}(t) \sum_{i=1}^{4}\left(1-\alpha_{i j} x_{j}(t)\right), \quad i=1,2,3,4 .
$$

As the diffusion matrix ' $g$ ' is dependent on the solution $X_{t}$, involved with the system (3.9), hence the system (3.5)-(3.8) is said to have multiplicative noise.

The main object of this paper is to analyze the effect of environmental fluctuations on the dynamic behavior of competitive system in presence of multiplicative white noise terms. Without any loss of generality we can assume, $\sigma_{1}>0, \sigma_{2}>0, \sigma_{3}>0, \sigma_{4}>0$. To investigate the dynamical behavior, first it is required to prove the global existence of the positive solution. Moreover, as we are dealing with a population dynamics model, we are concerned with non-negative solutions only. Hence in the next sub-section, we first show that the solution of the system (3.5)-(3.8) is positive and global.

\subsection{Existence and uniqueness of the positive global solution}

A stochastic differential equation system will have a unique global (i.e. no explosion in a finite time) solution for any given positive initial condition if the functions involved with the system satisfy the linear growth condition and local Lipschitz condition [51,52]. Moreover, one can easily check that all the coefficients of equations (3.5)-(3.8) do not satisfy the linear growth condition, though they are locally Lipschitz continuous, so the solution of equations (3.5)-(3.8) may explode at a finite time [53-55].

First we prove that there exists a unique positive local solution of the system (3.5)-(3.8), and then using the Lyapunov method [56], we prove that this solution is global. Explanation for the term 'explosion time' used in following lemma can be found in [51]. For the rest of this paper we introduce the following,

$$
\operatorname{Int}\left(\mathbb{R}_{+}^{4}\right)=\left\{\left(x_{1}, x_{2}, x_{3}, x_{4}\right) \in \mathbb{R}^{4}: x_{1}>0, x_{2}>0, x_{3}>0, x_{4}>0\right\} .
$$

Lemma 3.1.1 For $\left(x_{10}, x_{20}, x_{30}, x_{40}\right) \in \operatorname{Int}\left(\mathbb{R}_{+}^{4}\right)$, there is a unique positive local solution $\left(x_{1}(t), x_{2}(t), x_{3}(t), x_{4}(t)\right)$ of the system (3.5)-(3.8) for $t \in\left[0, \tau_{e}\right)$ almost surely(a.s.) where $\tau_{e}$ is the explosion time.

Proof: Using the transformation of variables $u_{i}(t)=\log x_{i}(t), i=1,2,3,4$ in (3.5)-(3.8) and applying Itô's formula we get,

$$
d u_{i}(t)=\left[\rho_{i}\left(1-\sum_{j=1}^{4} \alpha_{i j} e^{u_{j}(t)}\right)-\frac{\sigma_{i}^{2}}{2}\right] d t+\sigma_{i} d B_{i}(t)
$$


subjected to the initial condition $u_{i}(0)=\log x_{i 0}, i=1,2,3,4$. Clearly the coefficients of above stochastic differential system satisfy both linear growth condition and local Lipschitz condition. Hence there exist unique local solution $\left(u_{1}(t), u_{2}(t), u_{3}(t), u_{4}(t)\right)$ for the above stochastic system (3.10) for all $t \in\left[0, \tau_{e}\right)$. Clearly, $x_{i}(t)=e^{u_{i}(t)}, \quad i=1,2,3,4$, is the unique positive local solution of stochastic system (3.5)-(3.8) starting from an interior point of the positive cone. Now we show that this unique solution is not only a local solution rather it is global solution. To prove this we have to show $\tau_{e}=\infty$ a.s. We utilize the following lemma (see [56] for proof).

Lemma 3.1.2 For all $z>0$ the following inequality holds

$$
z \leq 2(z+1-\log z)-(4-2 \log 2) .
$$

Theorem 3.1.1 For any initial condition $X_{t 0} \equiv\left(x_{10}, x_{20}, x_{30}, x_{40}\right) \in \operatorname{Int}\left(\mathbb{R}_{+}^{4}\right)$, there exist unique solution $X_{t}$ for the stochastic system (3.5)-(3.8) for all $t \geq 0$.

Proof : We choose a sufficiently large non-negative number $m_{0}$ such that each of $x_{10}, x_{20}, x_{30}$ and $x_{40}$ stay within the interval $\left[\frac{1}{m_{0}}, m_{0}\right]$. For each integer $m \geq m_{0}$, we can define the stopping time

$$
\tau_{m}=\inf \left\{t \in\left[0, \tau_{e}\right): \min \left\{x_{1}(t), x_{2}(t), x_{3}(t), x_{4}(t)\right\} \leq \frac{1}{m} \text { or } \max \left\{x_{1}(t), x_{2}(t), x_{3}(t), x_{4}(t)\right\} \geq m\right\},
$$

where $\inf \emptyset=\infty$ ( $\emptyset$ denotes the empty set). Clearly, $\tau_{m}$ is increasing as $m \rightarrow \infty$. Set $\tau_{\infty}=\lim _{m \rightarrow \infty} \tau_{m}$ whence $\tau_{\infty} \leq \tau_{e}$ a.s. To prove that $\tau_{e}=\infty$, it is sufficient to prove that $\tau_{\infty}=\infty$ a.s. If possible, let us assume the statement be false. Then there exists two constants $T>0$ and $\epsilon \in(0,1)$ such that

$$
P\left\{\tau_{\infty} \leq T\right\}>\epsilon
$$

Hence we can find an integer $m_{1} \geq m_{0}$ such that

$$
P\left\{\tau_{m} \leq T\right\} \geq \epsilon
$$

for all $m \geq m_{1}$. Define a $C^{2}$-function $V: \operatorname{Int}\left(\mathbb{R}_{+}^{4}\right) \rightarrow \mathbb{R}_{+}$by,

$V\left(X_{t}\right)=\sum_{i=1}^{4}\left(x_{i}+1-\log x_{i}\right)$.

As $z+1-\log z>0$, for all $z>0$, the function $V\left(\right.$.) is positive definite for all $X_{t} \in \operatorname{Int}\left(\mathbb{R}_{+}^{4}\right)$. Calculating the differential of $V$ along the solution trajectories of the system (3.5)-(3.8) using Itô's formula, we get

$$
d V\left(X_{t}\right)=\left[\sum_{i=1}^{4} \rho_{i}\left(x_{i}-1\right)\left(1-\sum_{j=1}^{4} \alpha_{i j} x_{j}\right)+\sum_{i=1}^{4} \frac{\sigma_{i}^{2}}{2}\right] d t+\sum_{i=1}^{4} \sigma_{i}\left(x_{i}-1\right) d B_{i} .
$$

Positivity of $x_{i}(t)$ implies,

$$
\begin{aligned}
d V\left(x_{1}, x_{2}, x_{3}, x_{4}\right) & \leq\left[x_{1}\left(2 \rho_{1}+\rho_{2} \alpha_{21}+\rho_{3} \alpha_{31}+\rho_{4} \alpha_{41}\right)+x_{2}\left(\rho_{1} \alpha_{12}+2 \rho_{2}+\rho_{3} \alpha_{32}+\rho_{4} \alpha_{42}\right)\right. \\
& +x_{3}\left(\rho_{1} \alpha_{13}+\rho_{2} \alpha_{23}+2 \rho_{3}+\rho_{4} \alpha_{43}\right)+x_{4}\left(\rho_{1} \alpha_{14}+\rho_{2} \alpha_{24}+2 \rho_{3} \alpha_{34}+2 \rho_{4}\right) \\
& \left.+\frac{\sigma_{1}^{2}+\sigma_{2}^{2}+\sigma_{3}^{2}+\sigma_{4}^{2}}{2}\right] d t+\sum_{i=1}^{4} \sigma_{i}\left(x_{i}-1\right) d B_{i} .
\end{aligned}
$$

Defining six positive constants as follows

$$
c_{1}=\frac{\sigma_{1}^{2}}{2}+\frac{\sigma_{2}^{2}}{2}+\frac{\sigma_{3}^{2}}{2}+\frac{\sigma_{4}^{2}}{2},
$$




$$
\begin{gathered}
c_{2}=2 \rho_{1}+\rho_{2} \alpha_{21}+\rho_{3} \alpha_{31}+\rho_{4} \alpha_{41}, c_{3}=\rho_{1} \alpha_{12}+2 \rho_{2}+\rho_{3} \alpha_{32}+\rho_{4} \alpha_{42}, \\
c_{4}=\rho_{1} \alpha_{13}+\rho_{2} \alpha_{23}+2 \rho_{3}+\rho_{4} \alpha_{43}, c_{5}=\rho_{1} \alpha_{14}+\rho_{2} \alpha_{24}+2 \rho_{3} \alpha_{34}+2 \rho_{4}, \\
c_{6}=\max \left\{c_{2}, c_{3}, c_{4}, c_{5}\right\} .
\end{gathered}
$$

and using lemma (3.1.2), we can write

$$
c_{2} x_{1}+c_{3} x_{2}+c_{4} x_{3}+c_{5} x_{4} \leq 2 \sum_{j=1}^{4} c_{j+1}\left(x_{j+1}-\log x_{j}\right) \leq 2 c_{6} V\left(X_{t}\right) .
$$

Using (3.13) we can write,

$$
d V\left(X_{t}\right) \leq\left(c_{1}+2 c_{6} V\left(X_{t}\right)\right) d t+\sum_{j=1}^{4} \sigma_{j}\left(x_{j}-1\right) d B_{j}
$$

Finally assume, $c_{7}=\max \left\{c_{1}, 2 c_{6}\right\}$, and hence

$$
d V\left(X_{t}\right) \leq c_{7}\left(1+V\left(X_{t}\right)\right) d t+\sum_{j=1}^{4} \sigma_{j}\left(x_{j}-1\right) d B_{j}
$$

Therefore for $t_{1} \leq T$,

$$
\int_{0}^{\tau_{m} \wedge t_{1}} d V\left(X_{t}\right) \leq c_{7} \int_{0}^{\tau_{m} \wedge t_{1}}\left(1+V\left(X_{t}\right)\right) d t+\sum_{j=1}^{4} \sigma_{j} \int_{0}^{\tau_{m} \wedge t_{1}}\left(x_{j}-1\right) d B_{j} .
$$

where $\tau_{m} \wedge t_{1}=\min \left\{\tau_{m}, t_{1}\right\}$. Hence

$$
\begin{aligned}
V\left(x_{1}\left(\tau_{m} \wedge t_{1}\right), x_{2}\left(\tau_{m} \wedge t_{1}\right), x_{3}\left(\tau_{m} \wedge t_{1}\right), x_{4}\left(\tau_{m} \wedge t_{1}\right)\right) \leq & V\left(x_{10}, x_{20}, x_{30}, x_{40}\right) \\
& +c_{7} \int_{0}^{\tau_{m} \wedge t_{1}}\left(1+V\left(x_{1}, x_{2}, x_{3}, x_{4}\right)\right) d t \\
& +\sigma_{1} \int_{0}^{\tau_{m} \wedge t_{1}}\left(x_{1}-1\right) d B_{1}+\sigma_{2} \int_{0}^{\tau_{m} \wedge t_{1}}\left(x_{2}-1\right) d B_{2} \\
& +\sigma_{3} \int_{0}^{\tau_{m} \wedge t_{1}}\left(x_{3}-1\right) d B_{3} \sigma_{4} \int_{0}^{\tau_{m} \wedge t_{1}}\left(x_{4}-1\right) d B_{4} .
\end{aligned}
$$

Taking expectation of both sides of the above inequality, using the "mean zero" property of Itô's integral [57] and applying Fubini's theorem $[45,58,59]$ we get from the above inequality

$$
\begin{aligned}
E V\left(X_{\tau_{m} \wedge t_{1}}\right) & \leq V\left(X_{t 0}\right)+c_{7} E \int_{0}^{\tau_{m} \wedge t_{1}}\left(1+V\left(X_{t}\right)\right) d t \leq V\left(X_{t 0}\right)+c_{7} t_{1}+c_{7} E \int_{0}^{\tau_{m} \wedge t_{1}} V\left(X_{t}\right) d t \\
& \leq V\left(X_{t 0}\right)+c_{7} T+c_{7} E \int_{0}^{t_{1}} V\left(X_{\tau_{m} \wedge t}\right) d t=V\left(X_{t 0}\right)+c_{7} T+c_{7} \int_{0}^{\tau_{m} \wedge t_{1}} E V\left(X_{\tau_{m} \wedge t}\right) d t .
\end{aligned}
$$

Finally applying Gronwall's inequality [52], we get

$$
E V\left(X_{\tau_{m} \wedge T}\right) \leq c_{8}
$$

where

$$
c_{8}=\left(V\left(X_{t 0}\right)+c_{7} T\right) e^{c_{7} T} .
$$


Set

$$
\Omega_{m}=\left\{\tau_{m} \leq T\right\},
$$

for $m \geq m_{1}$. So by (3.12), we have $P\left(\Omega_{m}\right) \geq \epsilon$. Note that for every $\omega \in \Omega_{m}$, there is at least one of $x_{1}\left(\tau_{m}, \omega\right), x_{2}\left(\tau_{m}, \omega\right), x_{3}\left(\tau_{m}, \omega\right)$ and $x_{4}\left(\tau_{m}, \omega\right)$ which is equal to either $m$ or $\frac{1}{m}$ and hence $V\left(X_{\tau_{m}}\right)$ is no less than the smallest of $m+1-\log m$ and $\frac{1}{m}+1-\log \left(\frac{1}{m}\right)=\frac{1}{m}+1+\log m$. Consequently,

$$
V\left(X_{\tau_{m}}\right) \geq(m+1-\log m) \wedge\left(\frac{1}{m}+1+\log m\right) .
$$

Therefore from (3.11) and (3.14), it follows that

$$
c_{8} \geq E\left[1_{\Omega_{m}}(\omega) V\left(X_{\left(\tau_{m}, \omega\right)}\right)\right] \geq \epsilon\left[(m+1-\log m) \wedge\left(\frac{1}{m}+1+\log m\right)\right],
$$

where $1_{\Omega_{m}}$ is the indicator function of $\Omega_{m}$.

Making $m \rightarrow \infty$ we get $\infty>c_{5}=\infty$, which is a contradiction. Hence we must have $\tau_{\infty}=\infty$ a.s. This completes the proof of the above theorem.

\subsection{Moment boundedness}

Theorem 3.2.1: Let $\left(x_{1}(t), x_{2}(t), x_{3}(t), x_{4}(t)\right)$ be a solution of the system (3.5)-(3.8) for given initial value $\left(x_{10}, x_{20}, x_{30}, x_{40}\right) \in \operatorname{Int}\left(\mathbb{R}_{+}^{4}\right)$. Then we have the following

$$
\limsup _{t \rightarrow \infty} E\left[x_{i}^{p}(t)\right] \leq M_{i}(p), \quad i=1,2,3,4
$$

for all $p \geq 1$ where

$$
M_{i}(p)=\left[\frac{2 \rho_{i}+\sigma_{i}^{2}(p-1)}{2 \rho_{i}}\right]^{p}, \quad i=1,2,3,4
$$

Proof : Let $W\left(x_{1}(t)\right)=\left[x_{1}(t)\right]^{p}$ for $x_{1}(t) \in(0, \infty)$ and $p \geq 1$. Applying Ito's formula we get from (3.5),

$$
d W\left(x_{1}(t)\right)=\left[p x_{1}^{p} \rho_{1}\left(1-x_{1}-\alpha_{12} x_{2}-\alpha_{13} x_{3}-\alpha_{14} x_{4}\right)+\frac{1}{2} p(p-1) \sigma_{1}^{2} x_{1}^{p}\right] d t+\sigma_{1} p x_{1}^{p} d B_{1}(t) .
$$

Integrating both sides from 0 to $t$ we get,

$$
x_{1}^{p}(t)=x_{10}^{p}+\int_{0}^{t} p x_{1}^{p}(s)\left[\rho_{1}\left(1-x_{1}-\alpha_{12} x_{2}-\alpha_{13} x_{3}-\alpha_{14} x_{4}\right)+\frac{1}{2}(p-1) \sigma_{1}^{2}\right] d s+\int_{0}^{t} \sigma_{1} p x_{1}^{p}(s) d B_{1}(s) .
$$

Now taking expectation and using the "mean zero" property of Ito's integral, we can derive

$$
E\left[x_{1}^{p}(t)\right]=x_{10}^{p}+E \int_{0}^{t} p x_{1}^{p}(s)\left[\rho_{1}\left(1-x_{1}-\alpha_{12} x_{2}-\alpha_{13} x_{3}-\alpha_{14} x_{4}\right)+\frac{1}{2}(p-1) \sigma_{1}^{2}\right] d s
$$

Applying Fubini's theorem, we get

$$
\begin{aligned}
E\left[x_{1}^{p}(t)\right]= & x_{10}^{p}+p\left[\rho_{1}+\frac{\sigma_{1}^{2}}{2}(p-1)\right] \int_{0}^{t} E\left[x_{1}^{p}(s)\right] d s-p \rho_{1} \int_{0}^{t} E\left[x_{1}^{p+1}(s)\right] d s \\
& -p \rho_{1} \alpha_{12} \int_{0}^{t} E\left[x_{1}^{p}(s) x_{2}(s)\right] d s-p \rho_{1} \alpha_{13} \int_{0}^{t} E\left[x_{1}^{p}(s) x_{3}(s)\right] d s-p \rho_{1} \alpha_{14} \int_{0}^{t} E\left[x_{1}^{p}(s) x_{4}(s)\right] d s .
\end{aligned}
$$

Differentiating both sides with respect to $t$, we get

$$
\frac{d E\left[x_{1}^{p}(t)\right]}{d t}=p\left[\rho_{1}+\frac{\sigma_{1}^{2}}{2}(p-1)\right] E\left[x_{1}^{p}(t)\right]-p \rho_{1} E\left[x_{1}^{p+1}(t)\right]-p \rho_{1} \sum_{j=2}^{4} \alpha_{1 j} E\left[x_{1}^{p}(t) x_{j}(t)\right] .
$$


Using Holder's inequality,

$$
E\left[x_{1}^{p+1}(t)\right] \geq\left\{E\left[x_{1}^{p}(t)\right]\right\}^{\frac{p+1}{p}}
$$

we get from $(3.15)$

$$
\frac{d E\left[x_{1}^{p}(t)\right]}{d t} \leq p\left[\rho_{1}+\frac{\sigma_{1}^{2}}{2}(p-1)\right] E\left[x_{1}^{p}(t)\right]-p \rho_{1}\left\{E\left[x_{1}^{p}(t)\right]\right\}^{\frac{p+1}{p}} .
$$

One can easily verify that,

$$
u(t)=\left\{u_{0}^{-\frac{1}{\lambda}} e^{-\alpha t}+\frac{\beta}{\alpha}\left[1-e^{-\alpha t}\right]\right\}^{-\lambda}
$$

is the solution of ordinary differential equation

$$
\frac{d u(t)}{d t}=\lambda u(t)\left[\alpha-\beta u^{\frac{1}{\lambda}}(t)\right],
$$

subjected to the initial condition $u(0)=u_{0}$. Letting $t \rightarrow \infty$, we get

$$
u(t) \rightarrow\left[\frac{\alpha}{\beta}\right]^{\lambda},
$$

whenever $\alpha, \beta>0$. Using above result and comparison argument we get from (3.16),

$$
\limsup _{t \rightarrow \infty} E\left[x_{1}^{p}(t)\right] \leq\left[\frac{2 \rho_{1}+\sigma_{1}^{2}(p-1)}{2 \rho_{1}}\right]^{p}:=M_{1}(p) .
$$

Proceeding in a similar manner we get other desired results,

$$
\limsup _{t \rightarrow \infty} E\left[x_{j}^{p}(t)\right] \leq\left[\frac{2 \rho_{j}+\sigma_{j}^{2}(p-1)}{2 \rho_{j}}\right]^{p}:=M_{j}(p), \quad j=2,3,4 .
$$

\subsection{Stochastic Boundedness of the solution}

Definition 4.1 The solution of (3.5)-(3.8) is said to be stochastically bounded if for any $\epsilon>0$, there is a constant $H \equiv H_{\epsilon}$ such that for any $X_{t 0} \in \operatorname{Int}\left(\mathbb{R}_{+}^{4}\right)$, we have

$$
\limsup _{t \rightarrow \infty} P\left\{|X(t)|:=\sqrt{\left(x_{1}^{2}(t)+x_{2}^{2}(t)+x_{3}^{2}(t)+x_{4}^{2}(t)\right)} \leq H\right\} \geq 1-\epsilon .
$$

Now we show that the solution of system (3.5)-(3.8) is stochastically bounded. From (3.17)- (3.18) we get,

$$
\limsup _{t \rightarrow \infty} E\left[x_{1}^{p}(t)+x_{2}^{p}(t)+x_{3}^{p}(t)+x_{4}^{p}(t)\right] \leq \sum_{j=1}^{4} M_{j}(p):=B(p)
$$

which implies that there is a $T>0$, such that

$$
E\left[x_{1}^{p}(t)+x_{2}^{p}(t)+x_{3}^{p}(t)+x_{4}^{p}(t)\right] \leq 4 B(p), \forall t>T .
$$

Also note that $E\left[x_{1}^{p}(t)+x_{2}^{p}(t)+x_{3}^{p}(t)+x_{4}^{p}(t)\right]$ is continuous function of ' $t$ ', hence there is a $\tilde{B}(p)>0$ such that

$$
E\left[x_{1}^{p}(t)+x_{2}^{p}(t)+x_{3}^{p}(t)+x_{4}^{p}(t)\right] \leq \tilde{B}(p), \forall t \in[0, T] .
$$


Let $K(p)=\max \{4 B(p), \tilde{B}(p)\}$, then

$$
E\left[x_{1}^{p}(t)+x_{2}^{p}(t)+x_{3}^{p}(t)+x_{4}^{p}(t)\right] \leq K(p)<\infty, \forall t \in[0, \infty) .
$$

Taking supremum in the above inequality for $t \geq 0$, we obtain,

$$
\sup _{t \geq 0} E\left[x_{1}^{p}(t)+x_{2}^{p}(t)+x_{3}^{p}(t)+x_{4}^{p}(t)\right] \leq K(p)<\infty, \forall t \in[0, \infty), p \geq 1 .
$$

Observe that $|X(t)|^{p} \leq 4^{\frac{p}{2}}\left(x_{1}^{p}+x_{2}^{p}+x_{3}^{p}+x_{4}^{p}\right)$. Thus it follows from (3.21) that for any $t \geq 0$,

$$
E\left[|X(t)|^{p}\right] \leq 4^{\frac{p}{2}} E\left[x_{1}^{p}+x_{2}^{p}+x_{3}^{p}+x_{4}^{p}\right] \leq 4^{\frac{p}{2}} K(p) \equiv K_{1}(p)<\infty .
$$

Applying Tchebychev's inequality, we have that for $H>0$

$$
P\{|X(t)|>H\} \leq \frac{\sup _{t \geq 0} E\left[|X(t)|^{2}\right]}{H^{2}} \leq \frac{K_{1}(p)}{H^{2}} .
$$

Therefore by choosing $H$ sufficiently large, (3.19) follows.

\section{Stochastic permanence}

In contrary to the deterministic model of population biology, there is no unique definition for the stochastic system to show the persistence of each species involved with the system. Based upon the model under consideration and the type of stochasticity involved in the system, several authors have used various types of definition like stochastic permanence, strong persistence in mean, weak persistence in mean [60]. Now we recall the definition of stochastic permanence from [60].

Definition 4.1 Model (3.5)-(3.8) is said to be stochastically permanent if for any $\epsilon \in(0,1)$, there exists a pair of positive constants $\beta_{1} \equiv \beta_{1}(\epsilon)$ and $\chi \equiv \chi(\epsilon)$ such that for any initial value $X_{t 0} \in \operatorname{Int}\left(\mathbb{R}_{+}^{4}\right)$, the solution satisfy the following two conditions:

$$
\liminf _{t \rightarrow \infty} P\{|X(t)| \leq \chi\} \geq 1-\epsilon, \quad \liminf _{t \rightarrow \infty} P\left\{|X(t)| \geq \beta_{1}\right\} \geq 1-\epsilon .
$$

Theorem 4.1 If $\min \left\{\rho_{1}, \rho_{2}, \rho_{3}, \rho_{4}\right\}>\max _{i=1,2,3,4}\left\{\frac{\sigma_{i}^{2}}{2}\right\}$, then the model (3.5)-(3.8) is stochastically permanent.

Proof : We prove the Theorem in two steps. Firstly, we show that for arbitrary $\epsilon>0$, there exists a constant $\beta_{1}>0$ such that $\liminf _{t \rightarrow \infty} P\left\{|X(t)| \geq \beta_{1}\right\} \geq 1-\epsilon$.

Define

$$
V_{1}(X)=\frac{1}{x_{1}+x_{2}+x_{3}+x_{4}},
$$

for $X \in \operatorname{Int}\left(\mathbb{R}_{+}^{4}\right)$. Then Itô's formula gives

$$
d V_{1}(X)=\left\{-V_{1}^{2}(X)\left[\sum_{i=1}^{4} \rho_{i} x_{i}\left(1-\sum_{j=1}^{4} \alpha_{i j} x_{j}\right)\right]+V_{1}^{3}(x) \sum_{i=1}^{4} \sigma_{i}^{2} x_{i}^{2}\right\} d t-V_{1}^{2}(x) \sum_{i=1}^{4} \sigma_{i} x_{i} d B_{i}(t) .
$$

Now we assume $\min \left\{\rho_{1}, \rho_{2}, \rho_{3}, \rho_{4}\right\}>\max _{i=1,2,3,4}\left\{\frac{\sigma_{i}^{2}}{2}\right\}$, then we can choose a positive constant $r<1$, such that

$$
\min \left\{\rho_{1}, \rho_{2}, \rho_{3}, \rho_{4}\right\}-\max _{i=1,2,3,4}\left\{\frac{\sigma_{i}^{2}}{2}\right\}>\frac{r}{2} \max _{1 \leq i \leq 4}\left\{\sigma_{i}^{2}\right\}
$$


Define $V_{2}(X)=\left(1+V_{1}(X)\right)^{r}$. Making use of Itô's formula, we find

$$
\begin{aligned}
d V_{2}(X)= & r\left(1+V_{1}(X)\right)^{r-1} d V_{1}(X)+\frac{r(r-1)}{2}\left(1+V_{1}(X)\right)^{r-2} d\left(V_{1}(X)\right)^{2} \\
= & r\left(1+V_{1}(X)\right)^{r-2}\left\{-\left(1+V_{1}(X)\right) V_{1}^{2}(X)\left[\sum_{i=1}^{4} \rho_{i} x_{i}\left(1-\sum_{j=1}^{4} \alpha_{i j} x_{j}\right)\right]+V_{1}^{3}(X)\left(\sum_{i=1}^{4} \sigma_{i}^{2} x_{i}^{2}\right)\right. \\
& \left.+\frac{r+1}{2} V_{1}^{4}(X)\left(\sum_{i=1}^{4} \sigma_{i}^{2} x_{i}^{2}\right)\right\} d t-r\left(1+V_{1}(X)\right)^{r-1} V_{1}^{2}(X)\left(\sum_{i=1}^{4} \sigma_{i} x_{i} d B_{i}(t)\right) \\
= & r\left(1+V_{1}(X)\right)^{r-2} F(X) d t-r\left(1+V_{1}(X)\right)^{r-1} V_{1}^{2}(X)\left(\sum_{i=1}^{4} \sigma_{i} x_{i} d B_{i}(t)\right)
\end{aligned}
$$

where

$$
\begin{aligned}
F(X)= & -\left(1+V_{1}(X)\right) V_{1}^{2}(X)\left[\sum_{i=1}^{4} \rho_{i} x_{i}\left(1-\sum_{j=1}^{4} \alpha_{i j} x_{j}\right)\right]+V_{1}^{3}(X)\left(\sum_{i=1}^{4} \sigma_{i}^{2} x_{i}^{2}\right) \\
& +\frac{r+1}{2} V_{1}^{4}(X)\left(\sum_{i=1}^{4} \sigma_{i}^{2} x_{i}^{2}\right) .
\end{aligned}
$$

Now,

$$
\begin{aligned}
F(X) \leq & -V_{1}^{2}(x)\left(1+\frac{1}{\sum_{i=1}^{4} x_{i}}\right)\left(\sum_{i=1}^{4} \rho_{i} x_{i}\right)+V_{1}^{2}(X)\left(1+\frac{1}{\sum_{i=1}^{4} x_{i}}\right) \sum_{i=1}^{4}\left(\sum_{j=1}^{4} \rho_{i} \alpha_{i j} x_{j}\right) x_{i}+ \\
& \max _{i=1,2,3,4}\left(\sigma_{i}^{2}\right)\left(\sum_{i=1}^{4} x_{i}^{2}\right) \frac{1}{\left(\sum_{i=1}^{4} x_{i}\right)^{3}}+\frac{r+1}{2} \max _{i=1,2,3,4}\left(\sigma_{i}^{2}\right)\left(\sum_{i=1}^{4} x_{i}^{2}\right) \frac{1}{\left(\sum_{i=1}^{4} x_{i}\right)^{4}} \\
\leq & -V_{1}^{2}(X) \min \left\{\rho_{1}, \rho_{2}, \rho_{3}, \rho_{4}\right\}+\frac{r+1}{2} \max _{i=1,2,3,4}\left(\sigma_{i}^{2}\right) V_{1}^{2}(X) \\
& +a^{*}\left(1+V_{1}(X)\right) \frac{1}{\left(\sum_{i=1}^{4} x_{i}\right)^{2}}\left(\sum_{i=1}^{\frac{4}{2} x_{i}^{2}+2} \sum_{1 \leq i, j \leq 4} x_{i} x_{4}+2 x_{2} x_{4}+2 x_{3} x_{4}\right)+\max _{i=1,2,3,4}\left\{\sigma_{i}^{2}\right\} V_{1}(x) \\
\leq & -V_{1}^{2}(X)\left[\min \left\{\rho_{1}, \rho_{2}, \rho_{3}, \rho_{4}\right\}-\frac{r+1}{2} \max _{i=1,2,3,4}\left(\sigma_{i}^{2}\right)\right]+V_{1}(X)\left[a^{*}+\max _{i=1,2,3,4}\left(\sigma_{i}^{2}\right)\right]+a^{*},
\end{aligned}
$$

where

$$
a^{*}=\max _{1 \leq i, j \leq 4}\left\{\rho_{i} \alpha_{i j}\right\} .
$$

Substituting the above inequality into (4.1) we get,

$$
\begin{aligned}
d V_{2}(X(t)) & \leq r\left(1+V_{1}(X)\right)^{r-2}\left\{-V_{1}^{2}(X)\left[\min \left\{\rho_{1}, \rho_{2}, \rho_{3}, \rho_{4}\right\}-\frac{r+1}{2} \max _{i=1,2,3,4}\left(\sigma_{i}^{2}\right)\right]\right. \\
& \left.+V_{1}(X)\left[a^{*}+\max _{i=1,2,3,4}\left(\sigma_{i}^{2}\right)\right]+a^{*}\right\} d t \\
& -r\left(1+V_{1}(X)\right)^{r-1} V_{1}^{2}(X)\left(\sum_{i=1}^{4} \sigma_{i} x_{i} d B_{i}(t)\right) .
\end{aligned}
$$


Now we choose small positive number $\delta$ satisfying the following condition

$$
0<\frac{\delta}{r}<\min \left\{\rho_{1}, \rho_{2}, \rho_{3}, \rho_{4}\right\}-\frac{r+1}{2} \max _{i=1,2,3,4}\left(\sigma_{i}^{2}\right) .
$$

Define $V_{3}(X(t))=\exp \{\delta t\} V_{2}(X(t))$. Using Itô's formula we get

$$
\begin{aligned}
d V_{3}(X(t))= & \delta \exp \{\delta t\} V_{2}(X) d t+\exp \{\delta t\} d V_{2}(X) \\
\leq & \exp \{\delta t\}\left(1+V_{1}(X)\right)^{r-2}\left\{\delta\left(1+V_{1}(X)\right)^{2}-r V_{1}^{2}(X)\left[\min \left\{\rho_{1}, \rho_{2}, \rho_{3}, \rho_{4}\right\}-\frac{r+1}{2} \max _{i=1,2,3,4}\left(\sigma_{i}^{2}\right)\right]\right. \\
& \left.+r V_{1}(X)\left[a^{*}+\max _{i=1,2,3,4}\left(\sigma_{i}^{2}\right)\right]+r a^{*}\right\} d t \\
& -r \exp \{\delta t\}\left(1+V_{1}(X)\right)^{r-1} V_{1}^{2}(X)\left(\sum_{i=1}^{4} \sigma_{i} x_{i} d B_{i}(t)\right) \\
= & r \exp \{\delta t\}\left(1+V_{1}(X)\right)^{r-2}\left\{-V_{1}^{2}(X)\left[\min \left\{\rho_{1}, \rho_{2}, \rho_{3}, \rho_{4}\right\}-\frac{r+1}{2} \max _{i=1,2,3,4}\left(\sigma_{i}^{2}\right)-\frac{\delta}{r}\right]\right. \\
& \left.+V_{1}(X)\left[a^{*}+\max _{i=1,2,3,4}\left(\sigma_{i}^{2}\right)+2 \frac{\delta}{r}\right]+a^{*}+\frac{\delta}{r}\right\} d t \\
& -r \exp \{\delta t\}\left(1+V_{1}(X)\right)^{r-1} V_{1}^{2}(X)\left(\sum_{i=1}^{4} \sigma_{i} x_{i} d B_{i}(t)\right) \\
= & : \exp \{\delta t\} M(X) d t-r \exp \{\delta t\}\left(1+V_{1}(X)\right)^{r-1} V_{1}^{2}(X)\left(\sum_{i=1}^{4} \sigma_{i} x_{i} d B_{i}(t)\right)
\end{aligned}
$$

where

$$
\begin{array}{r}
M(X)=r\left(1+V_{1}(X)\right)^{r-2}\left\{-V_{1}^{2}(X)\left[\min \left\{\rho_{1}, \rho_{2}, \rho_{3}, \rho_{4}\right\}-\frac{r+1}{2} \max _{i=1,2,3,4}\left(\sigma_{i}^{2}\right)-\frac{\delta}{r}\right]\right. \\
\left.+V_{1}(X)\left[a^{*}+\max _{i=1,2,3,4}\left(\sigma_{i}^{2}\right)+2 \frac{\delta}{r}\right]+a^{*}+\frac{\delta}{r}\right\} .
\end{array}
$$

By virtue of the condition (4.2), we get

$$
M(X) \leq r\left(1+V_{1}(X)\right)^{r-1}\left[a^{*}+\max _{i=1,2,3,4}\left(\sigma_{i}^{2}\right)+2 \frac{\delta}{r}\right] .
$$

Since $r<1$, it can be easily seen that $M(X)$ has an upper bound in $\operatorname{Int}\left(\mathbb{R}_{+}^{4}\right)$, namely

$$
\Delta:=\sup _{X \in \operatorname{Int}\left(\mathbb{R}_{+}^{4}\right)} M(X)<+\infty .
$$

Therefore

$$
d V_{3}(X(t)) \leq \Delta \exp \{\delta t\} d t-r \exp \{\delta t\}\left(1+V_{1}(X)\right)^{r-1} V_{1}^{2}(X)\left(\sum_{i=1}^{4} \sigma_{i} x_{i} d B_{i}(t)\right) .
$$

Integrating both sides of the above inequality and then taking expectation we get,

$$
E\left[V_{3}(X(t))\right]=E\left[\exp \{\delta t\}\left(1+V_{1}(X)\right)^{r}\right] \leq\left(1+V_{1}\left(X_{0}\right)\right)^{r}+\frac{\Delta}{\delta} \exp \{\delta t\} .
$$

Then

$$
\limsup _{t \rightarrow \infty} E\left[V_{1}^{r}(X(t))\right] \leq \limsup _{t \rightarrow \infty} E\left[\left(1+V_{1}(X)\right)^{r}\right] \leq \frac{\Delta}{\delta}
$$


Now for $X(t) \in \operatorname{Int}\left(\mathbb{R}_{+}^{4}\right)$, we have

$$
\left(\sum_{i=1}^{4} x_{i}(t)\right)^{r} \leq 4^{r}\left(\max _{i=1,2,3,4} x_{i}(t)\right)^{r}=4^{r}\left(\max _{i=1,2,3,4} x_{i}^{2}(t)\right)^{0.5 r} \leq 4^{r}|X(t)|^{r} .
$$

Therefore

$$
\limsup _{t \rightarrow+\infty} E\left[\frac{1}{|X(t)|^{r}}\right] \leq 4^{r} \frac{\Delta}{\delta}:=\Delta_{1}
$$

Now for any $\epsilon>0$, choose $\beta_{1}=\frac{\epsilon^{\frac{1}{r}}}{\Delta_{1}^{\frac{1}{r}}}$. Therefore applying Tchebyshev's inequality, we get

$$
P\left\{|X(t)|<\beta_{1}\right\}=P\left\{\frac{1}{|X(t)|^{r}}>\frac{1}{\beta_{1}^{r}}\right\} \leq \beta_{1}^{r} E\left[\frac{1}{|X(t)|^{r}}\right],
$$

which implies that

$$
\limsup _{t \rightarrow+\infty} P\left\{|X(t)|<\beta_{1}\right\} \leq \beta_{1}^{r} \Delta_{1}=\epsilon
$$

Consequently,

$$
\liminf _{t \rightarrow+\infty} P\left\{|X(t)| \geq \beta_{1}\right\} \geq 1-\epsilon .
$$

Next it remains to prove that for arbitrary $\epsilon>0$, there exists $\chi>0$ such that $\liminf _{t \rightarrow+\infty} P(|X(t)| \leq \chi) \geq 1-\epsilon$. Proof of this part directly follows from the proof of stochastic boundedness.

\section{Numerical simulation results}

In this section, we provide some numerical simulation results to validate the analytical findings. Here we find the approximate strong solution to the stochastic system using the modified version of EulerMaruyama scheme with a correction term for stochastic increment.

All forthcoming numerical simulations are based upon the following stochastic differential system, for the specific choices of $s$ and $\sigma_{i}$ 's $i=1,2,3,4$,

$$
\begin{aligned}
& d x_{1}=\left[x_{1}\left(1-x_{1}-0.06 s x_{2}-2.2 s x_{3}-0.5 s x_{4}\right)\right] d t+\sigma_{1} x_{1} d W_{1}, \\
& d x_{2}=\left[0.42 x_{2}\left(1-0.36 s x_{1}-x_{2}-0.05 s x_{3}-1.32 s x_{4}\right)\right] d t+\sigma_{2} x_{2} d W_{2}, \\
& d x_{3}=\left[0.57 x_{3}\left(1-1.41 s x_{1}-1.07 s x_{2}-x_{3}-0.17 s x_{4}\right)\right] d t+\sigma_{3} x_{3} d W_{3}, \\
& d x_{4}=\left[0.72 x_{4}\left(1-0.26 s x_{1}-0.54 s x_{2}-1.15 s x_{3}-x_{4}\right)\right] d t+\sigma_{4} x_{4} d W_{4},
\end{aligned}
$$

and starting from the positive initial conditions. All numerical simulations reported here are carried out for the time step $\Delta t=0.01$ and it is worthy to mention here that the outcomes are verified with smaller time steps also. Our first intension is to show that the presence of small amplitude environmental driving force has the ability to alter the periodicity of the solutions compared to its deterministic counterpart. For $.7965 \leq s \leq .92915$, we have observed that the periodic solution bifurcating from coexisting equilibrium point and which is stable. Fig. 7 shows the time evolution of four competitors for $s=.9$ and $\sigma_{i}=.001$, $i=1,2,3,4$. In presence of small amplitude environmental fluctuation, we obtain the quasi-periodic oscillation for all species and this scenario persists for all values of $s<1$. From Fig. 7, the quasi-periodic nature is not very much clear but that can be understood from Fig. 8 where we have plotted $x_{1}$ against $x_{2}$ and a three dimensional plot of the same keeping the time on $x$-axis. Numerical simulation results for $s=.95$ and $s=.965$ with the same intensities of environmental fluctuation is presented in Fig. 9. It 


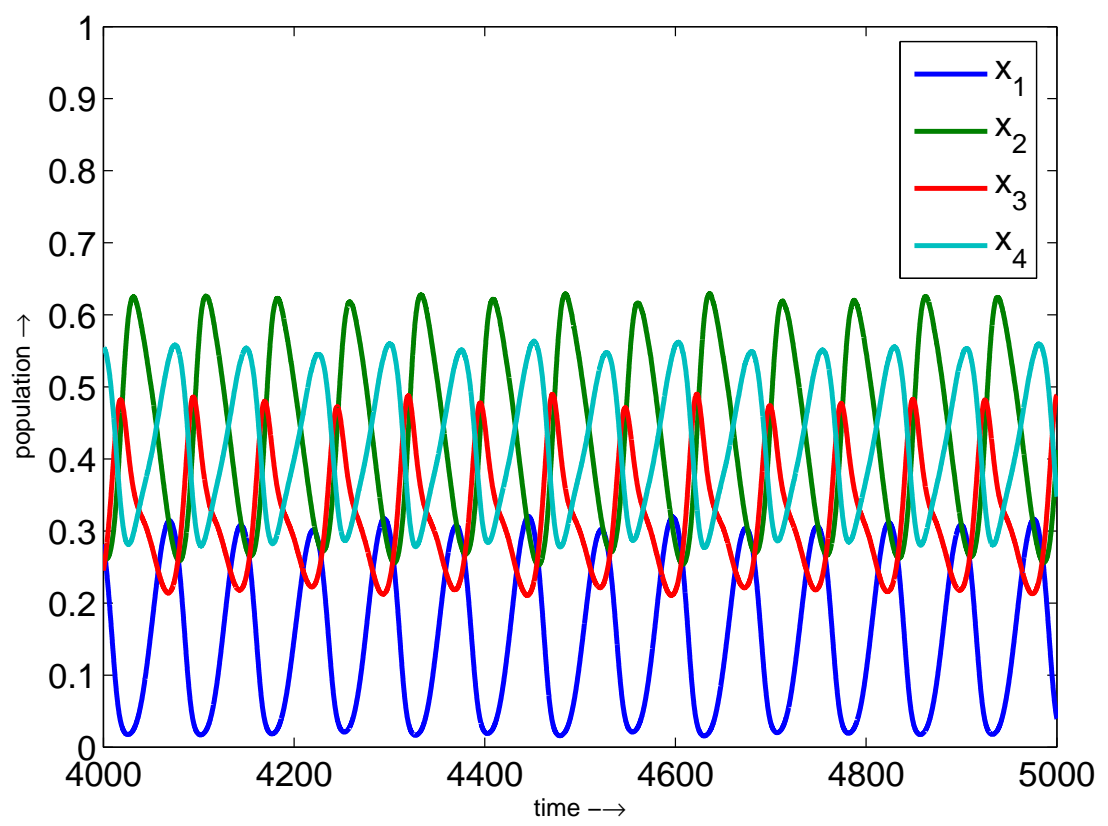

FIGURE 7. One sample path for each competitive species obtained through numerical simulation of the model (5.1) - (5.4) for $s=.9$ and $\sigma_{i}=.001, i=1,2,3,4$. Due to small noise strengths, solution trajectories are smooth.

is also interesting to note that the prominent stochasticity is not observed here for chosen magnitude of random forcing. With the same noise intensity, if we take $s=1.1$ then the solution trajectories are still quasi-periodic, but nature of quasi periodicity is little bit different from $s=.9$ (see Fig. 10). In case of small amplitude environmental noise, we obtain quasi-periodic oscillation for a wide range of values of $s$. It is important to mention here that the occurrence of peak or crush in population density may not occur at the same time point rather they vary from one simulation run to other. But basic feature of the dynamics should remain unaltered.

Next we consider the effect of large amplitude environmental fluctuation on the model under consideration. To do so, we consider the numerical simulation results for $s=.9$ and $\sigma_{i}=.2, i=1,2,3,4$. Fig. 11 shows the time evolution of all species which clearly shows that every component exhibit high amplitude non-equilibrium fluctuations and never they exhibit any stationary distributions. As the amplitude of environmental fluctuations satisfy the conditions required for the stochastic permanence, neither species goes to extinction. To validate the bounds of $p$-th order moments which is obtained in section 3 , we have calculated $E\left[x_{2}^{2}(t)\right]$ against time from one thousand simulation runs and obtained result is presented in Fig. 12. Simulation results are presented for $t \leq 5000$ and we have checked the result for longer time period also. It verify the analytical result $\lim _{\sup _{t \rightarrow \infty}} E\left[x_{2}^{2}(t)\right] \leq M_{2}(2)=1.045$. Interested readers can verify the same for other components as well as for other values of $p \geq 1$.

\section{Discussion}

The four species competitive model exhibit chaotic coexistence and this chaotic scenario arises through period doubling bifurcations. For a range of parameter values, the interior equilibrium point is locally asymptotically stable and then it becomes unstable through Hopf-bifurcation for $s>s_{1}=.7965$. The two periodic solution bifurcates from stable periodic attractor when the bifurcation parameter crosses it 

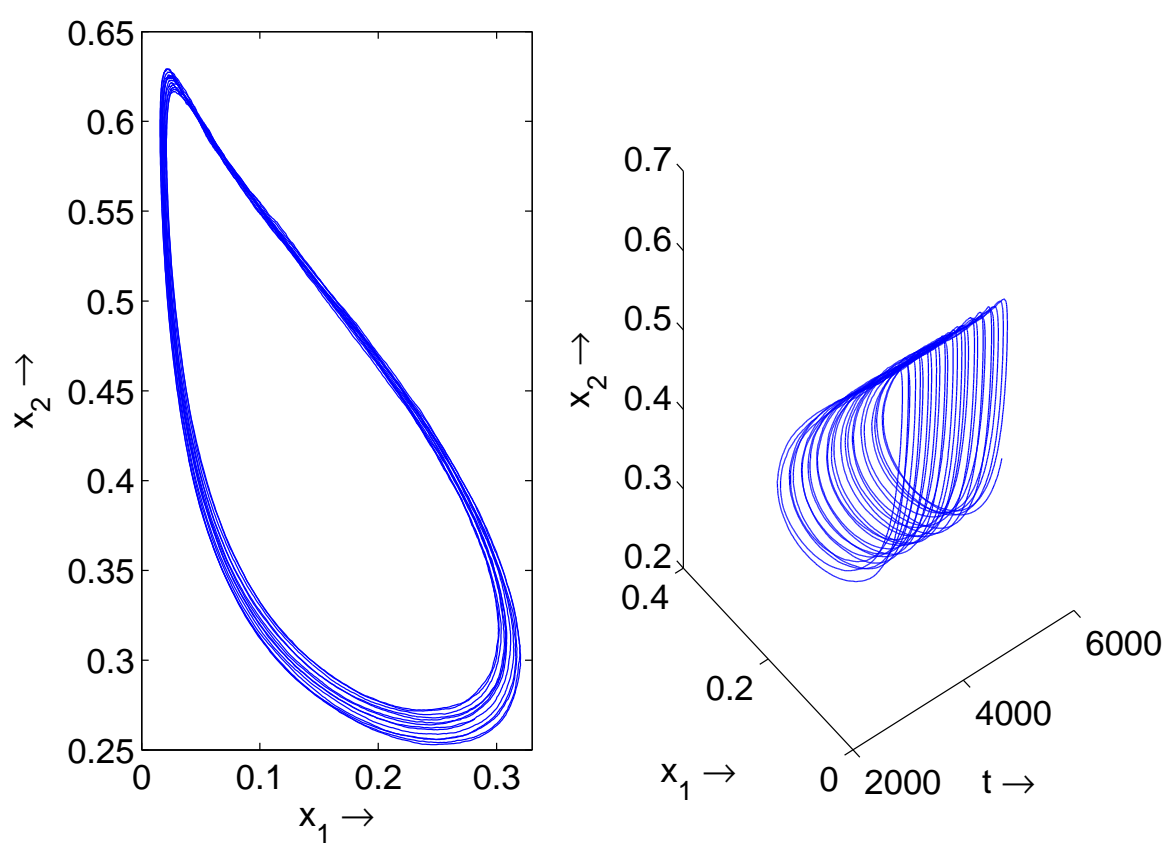

Figure 8. In the left panel the projection of quasi-periodic attractor on $x_{1} x_{2}$-plane is presented. Projection of solution trajectory on $t x_{1} x_{2}$-space is presented in the right panel. It clearly shows that the attractor in the phase-space in quasi-periodic.

threshold value $s_{2}=.92915$. As per deterministic analysis, the oscillatory coexistence state has period one just below the threshold $s_{2}$ and have period two just above it. But this type of sharp change in the periodicity of oscillation through a small perturbation into the system parameter is not a realistic feature. A sequence of period doubling takes place within the range $.92915<s<1.1$ leading to the chaotic oscillations for values of $s$ around 1.1. This type of sharp transition in periodicity of oscillation in population density is observed for the models considered within the deterministic setup, where neither the environmental stochasticity nor the demographic stochasticity is taken into account. It is true that we don't have unique modelling procedure where we can take care of all types of environmental driving forces. But one can construct stochastic model for the ecological system under consideration and compare the results obtained from the stochastic analysis with its deterministic counterpart.

In the present paper, we have constructed the stochastic model by perturbing the intrinsic growth rates of four competitive species with the help of mutually uncorrelated white noise terms. One can also raise the question that why the stochastic influence is not considered into the inter-specific as well as intra-specific competition coefficients. Answer to this question is that, it would be difficult to obtain analytical results with the help of available mathematical machinery. In this paper, we have proved the global existence of solution for the stochastic model and also obtained the bounds for $p$-th order moments of every species. The threshold magnitude of environmental forcing is derived to ensure the stochastic permanence, implying coexistence of each species at all future time. Numerical simulations are carried out to validate the analytical findings.

For the stochastic competitive model we have not observed any sharp transitions into the dynamics of the system when $s \in(.9,1.1)$ as we observed within the deterministic setup. Rather we found quasi-periodic oscillation for all species which sustain for a wide range of the parameter values of ' $s$ ' when magnitude of environmental fluctuations are small. Periodicity of the deterministic dynamics combined with the small amplitude environmental driving forces leads to the quasi-periodic oscillation. The 

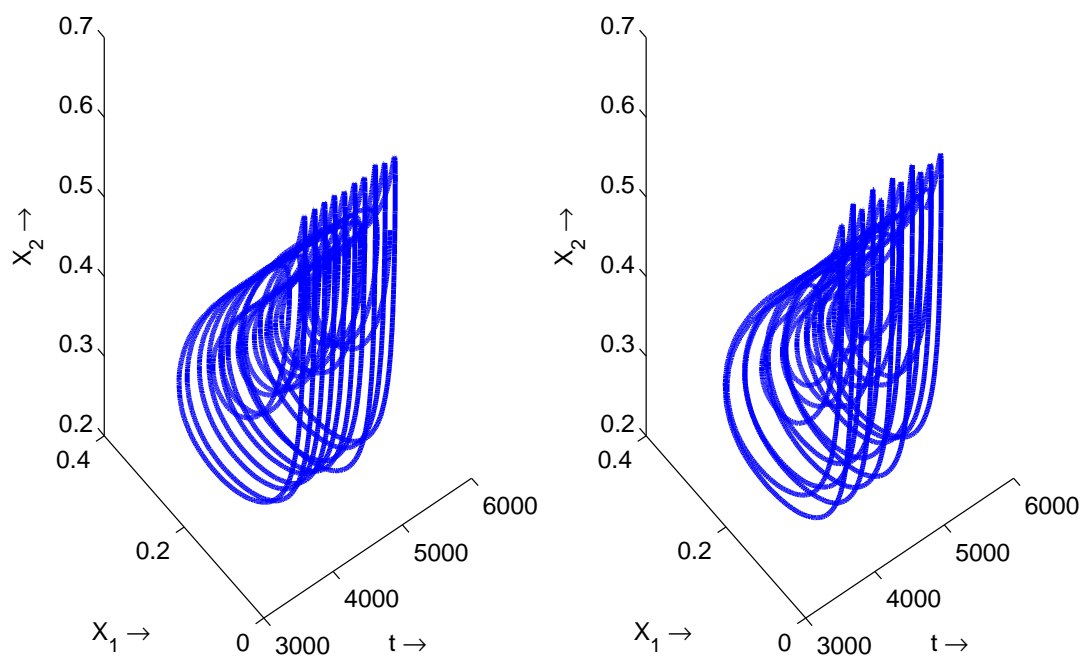

Figure 9. Projection of solution trajectory on $t x_{1} x_{2}$-space obtained for $s=.95$ (left panel) and $s=.965$ (right panel) with $\sigma_{i}=.001,(i=1,2,3,4)$.

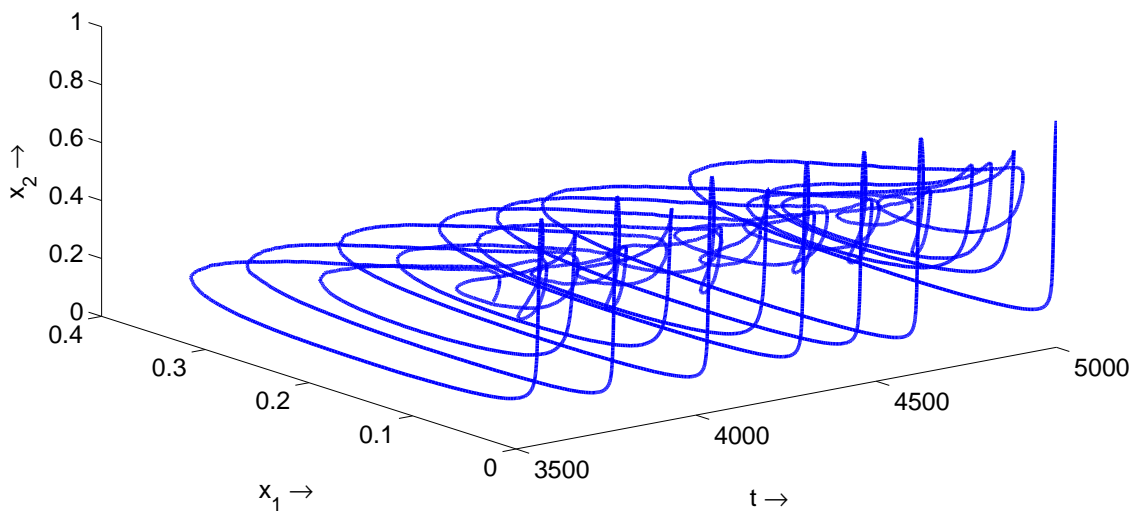

FiguRE 10. Projection of solution trajectory on $t x_{1} x_{2}$-space for $s=1.1$ and the noise strengths as mentioned in the caption of Fig. 7.

presence of continuously varying environmental stochasticity destroys completely the deterministic setup responsible for the successive period doubling leading to chaos. Further the amplitude of non-equilibrium fluctuations observed in each species when intensity of the environmental forcing is significantly high. The amplitude and rapidity of non-equilibrium fluctuation in population density is directly related to the magnitude of environmental driving forces. Our investigation also reveals that the extinction for 


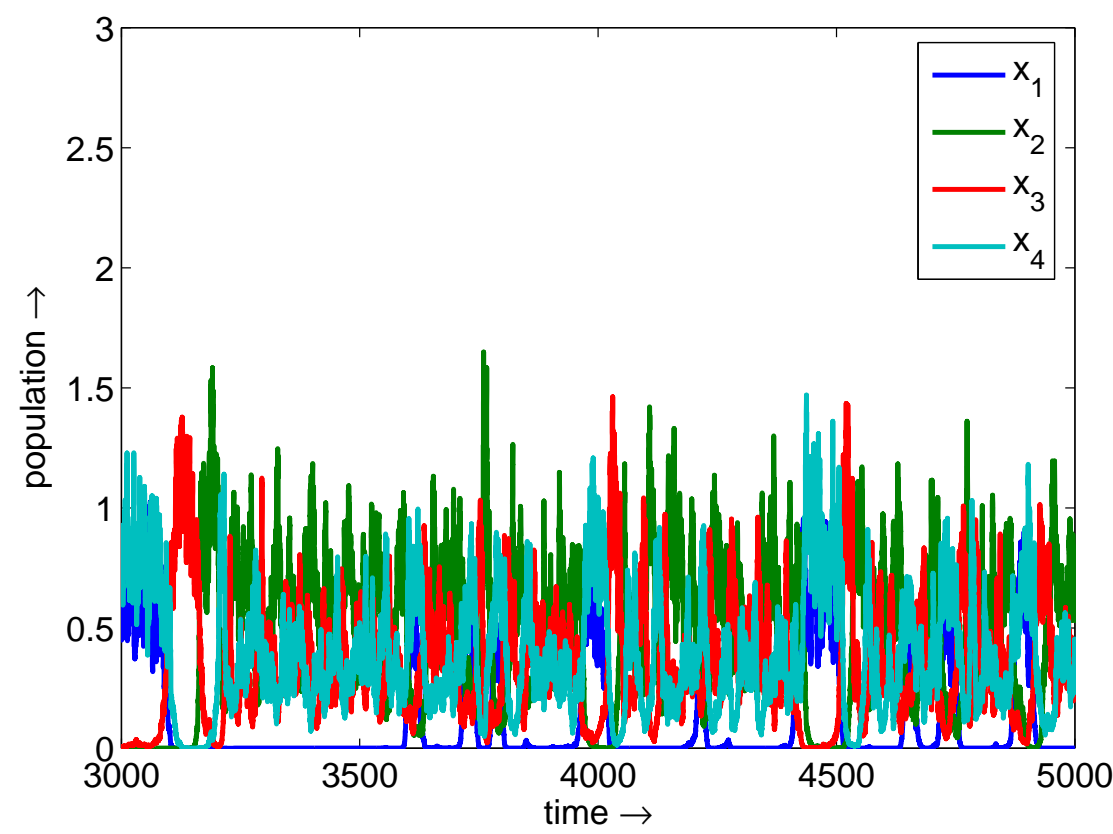

FiguRE 11. Time evolution of four competitive species obtained through one simulation run for $s=.9$ and $\sigma_{i}=.2, i=1,2,3,4$.

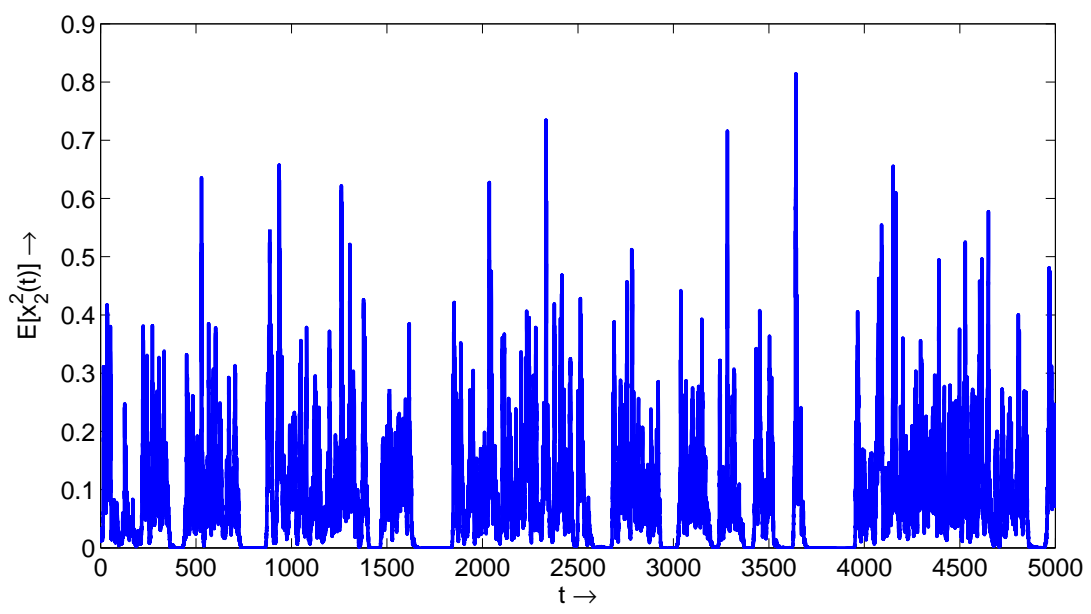

FiguRE 12. $E\left[x_{2}^{2}(t)\right]$ is calculated numerically for $0 \leq t \leq 5000$ by solving the stochastic model (5.1) - (5.4) 1000 times for $s=.9$ and $\sigma_{i}=.2, i=1,2,3,4$. Time evolution of $E\left[x_{2}^{2}(t)\right]$ shows it always remain below the limit $M_{2}(2)=1.045$

one or more competitive species is possible when the intensities of environmental forcing are very high. Finally, we like to remark that the validation of our analytical findings for realistic ecosystem demand the appropriate estimation for the parameters and strengths of environmental forcing along with the 
choice of appropriate time scale. In near future we will also investigate the same model by taking care of demographic stochasticity [61] for parameter values within and outside the chaotic regime.

Acknowledgements. The work of first author [P.S.M.] is supported by the Council of Scientific and Industrial Research, India. Authors are also thankful to the anonymous reviewer for his/her valuable suggestions that contributed to a significant improvement of the paper.

\section{References}

[1] B. Spagnolo, D. Valenti, A. Fiasconaro. Noise in ecosystems: A short review. Math. Bios. Eng., 1 (2004) $185-211$.

[2] R.M. May. Stability and complexity in Model Ecosystems. Princeton University Press, Princeton, NJ, USA, 1973.

[3] J.D. Murray, Mathematical Biology, Berlin, Springer-Verlag, 1989.

[4] E. Renshaw. Modelling Biological Populations in Space and Time. Cambridge University Press, Cambridge, 1993.

[5] P. Turchin. Complex Population Dynamics: A theoretical/empirical synthesis. Princeton, NJ: Princeton University Press, 2003.

[6] S. Smale. On the differential equations of species in competition. J. Math. Biol., 3 (1976) 5-7.

[7] S. Ellner, P. Turchin. Chaos in noisy world: new methods and evidence from time-series analysis. Am. Natur., 145 (1995) $343-375$.

[8] V. Volterra. Variazione e fluttuazione del numero d'individui in specie animali convinenti. Mem. Accad. Nazionale Lincei, 2 (1926) 31 - 113.

[9] A.J. Lotka . Elements of Mathematical Biology. New York, Dover, 1958.

[10] J.A. Vano, J.C. Wildenberg, M.B. Anderson, J.K. Noel, J.C. Sprott. Chaos in low-dimensional Lotka-Volterra Models of competition. Nonlinearity, 19 (2006) 2391-2404.

[11] R. Wang, D. Xiao. Bifurcations and chaotic dynamics in a 4-dimensional competitive Lotka-Volterra system. Nonlin Dyn, 59 (2010) 411- 422.

[12] E.C. Zeeman, M.L. Zeeman. An n-dimensional competitive Lotka-Volterra system is generically determined by the edges of its carrying simplex. Nonlinearity , 15 (2002) 2019-2032.

[13] M.W. Hirsch. Systems of differential equations which are competitive or cooperative III: Competing species. Nonlinearity 1 (1988) 51-71.

[14] M.W. Hirsch. Systems of differential equations which are competitive or cooperative V: Convergence in 3-dimensional systems. J. Differ. Equ., 80 (1989) 94-106.

[15] M.W. Hirsch. Systems of differential equations which are competitive or cooperative IV: Structural stability in three dimensional systems. SIAM J. Math. Anal., 21 (1990) 1225-1234.

[16] M.L. Zeeman. Hopf bifurcations in competitive three dimensional Lotka-Volterra systems. Dyn. Stab. Sys., 8 (1993) 189-217.

[17] M. Gyllenberg, P. Yan, Y. Wang. A 3D competitive Lotka-Volterra system with three limit cycles: A falsification of a conjecture by Hofbauer and So. Appl. Math. Lett., 19 (2006) 1-7.

[18] J. Hofbauer, J.W.H. So. Multiple limit cycles for three dimensional Lotka-Volterra equations. Appl. Math. Lett., 7 (1994) 65-70.

[19] Z. Lu, Y. luo. Three limit cycles for a three-dimensional Lotka-Volterra competitive system with a heteroclinic cycle Comput. Math. Appl., 46 (2003) 231-238.

[20] R.M. May, W.J. Leonard. Nonlinear aspects of competition between three species. SIAM J. Appl. Math., 29 (1975) 243-253.

[21] P.V.D. Driessche, M.L. Zeeman. Three-dimensional competitive Lotka-Volterra systems with no periodic orbits. SIAM J. Appl. Math., 58 (1998) 227-234.

[22] D. Xiao, W. Li. Limit cycles for the competitive three dimensional Lotka-Volterra systems. J. Differ. Equ., 164 (2000) $1-15$.

[23] A. Arneodo, P. Coullet, C. Tresser. Occurrence of strange attractors in three-dimensional Volterra equations. Phys. Lett. A, 79 (1980) 259-263.

[24] A. Arneodo, P. Coullet, J. Peyraud, C. Tresser. Strange attractors in Volterra equations for species in competition. J. Math. Biol., 14 (1982) 153-157.

[25] A. Hastings, T. Powell. Chaos in a three-species food chain. Ecology, 72 (1991), 896 - 903.

[26] A. Klebanoff, A. Hastings. Chaos in three species food chains. J. Math. Biol., 32 (1994), 427-451.

[27] K. McCann, P. Yodzis. Biological conditions for chaos in a three-species food chain. Ecology, 75 (1994), 561-564.

[28] D. V. Vayenas, S. Pavlou. Chaotic dynamics of a microbial system of coupled food chains. Ecol. Model., 136 (2001), 285-295.

[29] S. Abbas, D. Bahuguna, M. Banerjee. Effect of stochastic perturbation on a two species competitive model. Non. Anal. Hyb. Syst., 3 (2009) $195-206$.

[30] S.L. Pimm. The balance of nature? Ecological issue in the conservation of species and communities. University of Chicago Press, Chicago, 1991.

[31] J.H. Steele. A comparison of terrestrial and marine ecological systems. Nature, 313 (1985) 355 - 358. 
[32] P.S. Mandal, M. Banerjee. Stochastic persistence and stationary distribution in a Holling-Tanner type prey-predator model. Physica A, 391 (2012) 1216 - 1233.

[33] D. Nyccka, S. Ellner, D. Mccaffrey, A.R. Gallant. Finding chaos in noisy systems. J. Roy. Statist. Soc. B., 54 (1992) $399-426$.

[34] M. Bandyopadhyay. Effect of environmental fluctuation on a detritus based Ecosystem. J. Appl. Math. Comput., 26 (2008) $433-450$.

[35] D. Valenti, A. Flasconaro, B. Spagnolo. Stochastic resonance and noise delayed extinction in a model of two competing species. Physica. A., 331 (2004) $477-486$.

[36] N.V. Agudov, B. Spagnolo. Noise-enhanced stability of periodically driven metastable states. Phys. Rev. E., 64 (2001) 035102(R).

[37] C.V.D. Broeck, J.M.R. Parrondo, R. Toral, R. Kawai. nonequilibrium phase transitions induced by multiplicative noise. Phys. Rev. E., 55 (1997) $4084-4094$.

[38] H. Berry. Nonequilibrium phase transition in a self-activated biological network. Phys. Rev. E., 67 (2003) 031907.

[39] J. Li, P. Hanggi,Spatially periodic stochastic system with infinite globally coupled oscillators. Phys. Rev. E., 64 (2001) 011106.

[40] R. Arditi, L.R. Ginzburg. Coupling in predator-prey dynamics: ratio-dependence. J. Theor. Biol., 139 (1989) 311 326.

[41] M. Bandyopadhyay, R. Bhattacharya, C.G. Chakrabarti. A nonlinear two species oscillatory system: Bifurcation and stability analysis. Int. J. Math. Sci., (2003) 1981-1991.

[42] C.S. Elton. The pattern of Animal Communities. London, Methuen, 1966.

[43] W. Horsthemke, R. Lefever. Noise Induced Transitions. Springer-Verlag, Berlin, 1984.

[44] C.W. Gardiner. Handbook of Stochastic Methods. Springer-Verlag, New York, 1983.

[45] T.C.Gard. Introduction to Stochastic Differential Equations. Marcel Decker, New York, 1987.

[46] I.I. Gikhman, A.V. Skorokhod. The Theory of Stochastic Process-I. Berlin, Springer, 1979.

[47] X. Mao, G. Marion, E. Renshaw. Environmental Brownian noise suppresses explosions in population dynamics. Stoc. Proc. Appl., 97 (2002) 95 - 110.

[48] D.J. Higham. An algorithmic introduction to numerical simulation of stochastic differential equations. SIAM Rev., 43 (2001) $525-546$.

[49] V.B. Kolmanovskii, L.E. Shaikhet. Some peculiarities of the general method of Lyapunov functionals construction. Appl. Math. Lett., 15 (2002) 355 - 360.

[50] V.B. Kolmanovskii, L.E. Shaikhet. Construction of Lyapunov functionals for stochastic hereditary systems: a survey of some recent results. Math. Comp. Model., 36 (2002) 691 - 716.

[51] L. Arnold. Stochastic Differential Equations: Theory and Applications. Wiley, New York, 1972.

[52] X. Mao. Stochastic Differential Equations and Applications. Horwood, New York, 1997.

[53] R.Z. Khasminskii. Stochastic Stability of Differential Equations. Sijthoff and Noordhoff, Alphen a/d Rijn, 1981.

[54] X. Mao. Stability of Stochastic Differential Equations with respect to Semimartingales. Longman Scientific and Technical, New York, 1991.

[55] X. Mao. Exponential Stability of Stochastic Differential Equations. Marcel Dekker, New York, 1994.

[56] N. Dalal, D. Greenhalgh, X. Mao. A stochastic model for internal HIV dynamics. J. Math. Anal. Appl., 341 (2008) $1084-1101$

[57] E. Allen. Modeling With Itô Stochastic Differential Equations. Dordrecht, The Netherlands, 2007.

[58] V. Hutson, J.S. Pym. Applications of Functional Analysis and Operator Theory. Academic Press, London, 1980.

[59] A.N. Kolmogorov, S. V. Fomin. Introductory Real Analysis. Dover Publications, Inc., New York, 1970.

[60] M. Liu, K. Wang, Q. Wu. Survival analysis of stochastic competitive models in a polluted environment and stochastic competitive exclusion principle. Bull. Math. Biol., 73 (2011) 1969-2012.

[61] P.S. Mandal, M. Banerjee. Deterministic Chaos vs. Stochastic Fluctuation in an Eco-epidemic Model. Math. Model. Nat. Phenom., (In press), (2012). 